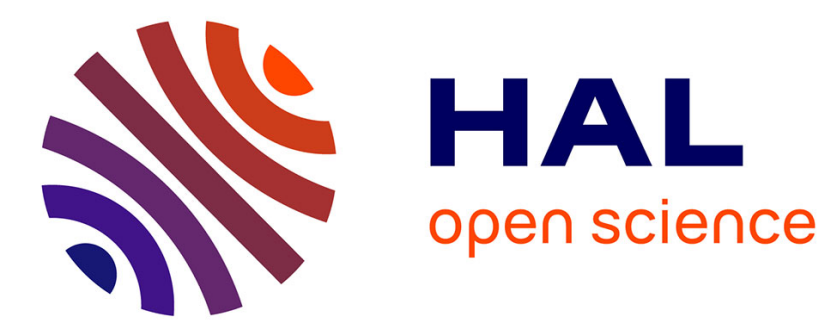

\title{
Experimental simulation of turbulent boundary layer induced vibrations by using a synthetic array
}

\author{
Mathieu Aucejo, Laurent Maxit, J.-L. Guyader
}

\section{To cite this version:}

Mathieu Aucejo, Laurent Maxit, J.-L. Guyader. Experimental simulation of turbulent boundary layer induced vibrations by using a synthetic array. Journal of Sound and Vibration, 2012, 331 (16), pp.3824-3843. 10.1016/j.jsv.2012.04.010 . hal-01668987

\section{HAL Id: hal-01668987 https://hal.science/hal-01668987}

Submitted on 14 Mar 2019

HAL is a multi-disciplinary open access archive for the deposit and dissemination of scientific research documents, whether they are published or not. The documents may come from teaching and research institutions in France or abroad, or from public or private research centers.
L'archive ouverte pluridisciplinaire HAL, est destinée au dépôt et à la diffusion de documents scientifiques de niveau recherche, publiés ou non, émanant des établissements d'enseignement et de recherche français ou étrangers, des laboratoires publics ou privés. 


\title{
Experimental simulation of Turbulent Boundary Layer induced vibrations by using a synthetic array
}

\author{
M. Aucejo ${ }^{\mathrm{a}}$, L. Maxit ${ }^{\mathrm{b}}$, J.-L. Guyader ${ }^{\mathrm{b}}$ \\ ${ }^{a}$ Structural Mechanics and Coupled Systems Laboratory, Conservatoire National des Arts \\ et Métiers, 2 Rue Conté, 75003 Paris, France \\ ${ }^{b}$ Laboratoire Vibrations Acoustique, INSA Lyon, 25 bis avenue Jean Capelle 69621 \\ Villeurbanne Cedex, FRANCE
}

\begin{abstract}
Of particular interest is the experimental feasibility of replacing wind tunnel measurements with a substitute experiment to simulate Turbulent Boundary Layer (TBL) induced vibrations. This paper describes the development of an experimental procedure to address this issue. The proposed approach is based on the concept of uncorrelated wall pressure plane waves, which is introduced as a prelude to this study. Concretely, an array of acoustic monopoles can be used to generate these wall pressure plane waves. To this end, the design parameters of the array are studied numerically. However, when dealing with experimental applications, the number of monopoles required being prohibitive, the principle of synthetic array is applied instead. This technique allows simulating the effect of an array of acoustic monopoles from sequential measurements. To assess the validity of the proposed approach, the whole procedure is applied to simulate TBL induced vibrations of a thin elastic plate. The results obtained are in good agreement with those
\end{abstract}

*Corresponding author. E-mail address : mathieu.aucejo@cnam.fr 
obtained from random vibration theory.

Keywords: Synthetic array, Turbulent Boundary Layer, Plane waves

\section{Introduction}

The study of Turbulent Boundary Layer (TBL) induced vibrations is of great importance in numerous industrial applications, especially when considering automotive and aeronautical applications or self-noise phenomena in underwater acoustics. Consequently, knowledge and understanding of physical mechanisms governing TBL induced vibrations is a crucial issue for engineers. From the practical point of view, in situ measurements, e.g. flight tests $[1,2,3,4]$, underwater measurements $[5,6]$ and wind tunnel measurements $[7,8]$ are generally used. However, these experimental set-ups are expensive due to the equipment and resources needed. Futhermore, measurements are difficult to carry out because background noise levels are generally high. It is thus of considerable interest to develop substitute experiments to assess TBL induced vibrations under laboratory conditions. Nevertheless, very few studies have been carried out in the last few decades. One of the first studies on this topic was proposed by Fahy in 1966. In [9], Fahy mentioned different ways of simulating TBL wall pressure fluctuations such as arrays of shakers or loudspeakers, although he could not verify these approaches experimentally because of the practical difficulties of implementing these solutions at that time.

Two decades later, Robert [10] studied the feasibility of using an array of suitably driven shakers by noting that only a limited number of structural modes are excited independently by the TBL. The main advantage of this 
approach is that the location of the shakers is independent of the convection velocity of the TBL, but as mentioned by Elliot et al. [11], this approach requires knowing the structural mode shapes to determine the number and the location of the shakers from a numerical process. Consequently, this implies their measurement by performing a preliminary modal analysis. Another semi-experimental approach, based on the standard formulation of random vibration theory [12] and the measurements of the required transfer functions, was proposed by Audet et al. [13] to assess hydrodynamic self-noise under a SONAR dome. Finally, the experimental real-time synthesis of spatially correlated random pressure fields such as TBL with a nearfield array of appropriately-driven loudspeakers was studied by Elliot et al. [11] and Maury and Bravo $[14,15,16]$. However, this approach, based on signal processing techniques, leads to the use of a great number of loudspeakers as frequency increases.

In the present paper, we propose an alternate off-line methodology using a single acoustic source to investigate structural vibrations induced by random pressure fields such as diffuse field, rain-on-the-roof or TBL excitations. Here, we focus more specifically on the TBL excitation, because of the industrial demand for cutting experimental cost associated with in situ or wind tunnel measurements. The aim is to overcome the difficulty of generating TBL excitation experimentally and gain the practical advantage of performing a substitute experiment based on acoustic excitation. For this purpose, the methodology developed in this article is based on three main features: (1) the wall pressure plane wave expansion to represent the target 
random wall-pressure field, (2) the identification steps to simulate TBL induced vibrations from individual transfer function measurements and (3) the concept of synthetic array. Although each of these techniques are separately well-known in the field of acoustics, the originality of this paper lies in their combined use to experimentally simulate the response of a structure excited by a random wall-pressure field such as TBL.

To simulate TBL induced vibrations from uncorrelated wall pressure plane waves, the amplitude of each wall pressure plane wave must be defined so that the whole set can represent the statistical properties of a wall pressure field generated by a TBL. This relation between wall pressure plane waves and TBL wall pressure fluctuations is first established in section 2 . The experimental implementation of this formulation implies the generation of wall pressure fields corresponding to those of propagating and evanescent acoustic plane waves in the direction normal to the observation area. A single acoustic source being not sufficient to generate such sound fields over the observation area, an array of acoustic monopoles is used. The design parameters of the array, such as the number of monopoles, its distance from the observation area and its size are then studied numerically and compared with existing theoretical and empirical criteria $[11,14,15]$ in section 3. However, the use of a full monopole array would be experimentally impossible at high frequencies, since it is necessary to increase the number of monopoles as frequency increases. Therefore a synthetic array is used instead to avoid such frequency limitations, and is the original development described herein. This technique permits using a single acoustic source to simulate the effect of an 
array composed of a large number of acoustic monopoles from a sequential process, a possibility allowed by the linear nature of the problem. The principle of the synthetic array and the corresponding formalism are presented in section 4. In section 5, an experimental validation of this procedure is proposed for a thin elastic plate. Two validation steps are presented: (1) reconstructions of wall pressure plane waves using a synthetic array and (2) comparison of TBL induced vibrations with a classical formulation when the response of the plate is calculated analytically. Finally, a complete implementation of the method is performed to demonstrate its versatility vis-a-vis the excitation model.

\section{Theoretical framework}

\subsection{Basic concept: The uncorrelated wall pressure plane waves}

As a random process, the wall pressure fluctuations of a TBL can only be modelled by using adapted statistical tools. Under the assumptions of stationarity and homogeneity, TBL excitation is thus represented by its crossspectral density (CSD) function. In the literature, numerous models are available, such as Corcos-like [17, 18, 19, 20] or Chase-like [21, 22, 23, 24] models, for describing TBL wall pressure fluctuations on a rigid plane. These models can be then directly used to determine the statistical properties of the response of a structure, such as a panel, thanks to the random vibration theory $[25,26]$.

Here, the basic idea is to represent both the TBL CSD function and the induced structural response as a combination of uncorrelated wall pressure 
plane waves. This approach is related to room acoustics, where a diffuse field can be approximated by summing the effect of an infinite number of acoustic plane waves originating from all spatial directions and having the same amplitude [27]. The term wall pressure plane waves refers to the TBL wall pressure field defined as the blocked pressure acting on a structure. Thus, a wall pressure plane wave is a surface wave, whose amplitude is scaled on the TBL CSD function. From this definition, the general form of a wall pressure plane wave $P_{r s}$ of wavenumbers $\left(k_{r}, k_{s}\right)$ is:

$$
P_{r s}(x, y, t)=A_{r s}(t) \mathrm{e}^{j k_{r} x+j k_{s} y}
$$

where $A_{r s}(t)$ is a random variable corresponding to the amplitude of a wall pressure plane wave of wavenumbers $\left(k_{r}, k_{s}\right)$.

The corresponding CSD function $S_{P_{r s} P_{r s}}$ between 2 points is therefore:

$$
S_{P_{r s} P_{r s}}\left(\xi_{x}, \xi_{y}, \omega\right)=S_{A_{r s} A_{r s}}(\omega) \mathrm{e}^{j k_{r} \xi_{x}+j k_{s} \xi_{y}}
$$

where $\xi_{x}$ and $\xi_{y}$ are the spatial shifts between 2 points along x-axis and yaxis respectively, $S_{A_{r s} A_{r s}}(\omega)$ is the auto-spectral density (ASD) function of the wall pressure plane wave amplitude and $\omega$ is the angular frequency.

Let us suppose now a rigid surface impacted by a set of uncorrelated wall pressure plane waves $P_{r s}$. The total pressure $p(x, y, t)$ at point $(\mathrm{x}, \mathrm{y})$ of the rigid surface is thus given by: 


$$
p(x, y, t)=\sum_{r, s} P_{r s}(x, y, t)
$$

As the wall pressure plane waves are supposed uncorrelated, $S_{A_{r s} A_{r^{\prime} s^{\prime}}}=0$ if $r \neq r^{\prime}$ and $s \neq s^{\prime}$. Consequently, the CSD function of the total pressure is:

$$
S_{p p}\left(\xi_{x}, \xi_{y}, \omega\right)=\sum_{r, s} S_{A_{r s} A_{r s}}(\omega) \mathrm{e}^{j k_{r} \xi_{x}+j k_{s} \xi_{y}}
$$

From this equation, it is now possible to model the TBL CSD function and the corresponding induced vibrations from uncorrelated wall pressure plane waves, as demonstrated in the next section.

\subsection{Modeling of the TBL excitation}

The CSD function of the TBL wall pressure fluctuations $S_{p p}\left(\xi_{x}, \xi_{y}, \omega\right)$ in physical space is related to that in the wavenumber-frequency space, i.e. $\Gamma_{p p}\left(k_{x}, k_{y}, \omega\right)$, by the following inverse spatial Fourier Transform:

$$
S_{p p}\left(\xi_{x}, \xi_{y}, \omega\right)=\iint_{-\infty}^{+\infty} \frac{\Gamma_{p p}\left(k_{x}, k_{y}, \omega\right)}{4 \pi^{2}} \mathrm{e}^{j k_{x} \xi_{x}+j k_{y} \xi_{y}} \mathrm{~d} k_{x} \mathrm{~d} k_{y}
$$

where $k_{x}$ and $\xi_{x}$ are the wavenumber and the spatial shift along the streamwise direction (x-axis) respectively, $k_{y}$ and $\xi_{y}$ are the wavenumber and the spatial shift along the spanwise direction (y-axis) respectively and $\omega$ is the angular frequency.

The discretization of the integration domain transforms Eq. (5) into its discrete form: 


$$
S_{p p}\left(\xi_{x}, \xi_{y}, \omega\right)=\sum_{r, s} \frac{\Gamma_{p p}\left(k_{r}, k_{s}, \omega\right)}{4 \pi^{2}} \mathrm{e}^{j k_{r} \xi_{x}+j k_{s} \xi_{y}} \Delta k_{r} \Delta k_{s}
$$

where the couple $\left(k_{r}, k_{s}\right)$ is the discrete analogue of the couple of wavenumbers $\left(k_{x}, k_{y}\right)$ and the couple $\left(\Delta k_{r}, \Delta k_{s}\right)$ defines the wavenumber resolution.

Direct comparison of Eq. (6) with Eq. (4) allows demonstrating that TBL excitation can be represented as a superposition of uncorrelated wall pressure plane waves, if the ASD function $S_{A_{r s} A_{r s}}$ of each wall pressure plane wave satisfies:

$$
S_{A_{r s} A_{r s}}(\omega)=\frac{\Gamma_{p p}\left(k_{r}, k_{s}, \omega\right) \Delta k_{r} \Delta k_{s}}{4 \pi^{2}}
$$

\subsection{Modeling of TBL induced vibrations}

From the previous analysis, it can be also shown that the ASD function of the velocity $S_{v v}(Q, \omega)$ at point $\mathrm{Q}$ of a structure under TBL excitation can be obtained from an uncorrelated wall pressure plane waves expansion. Indeed, if the structure is linear, the structural velocity ASD function can be expressed by a wave-vector integral $[25,28]$ :

$$
S_{v v}(Q, \omega)=\iint_{-\infty}^{+\infty} \frac{\Gamma_{p p}\left(k_{x}, k_{y}, \omega\right)}{4 \pi^{2}}\left|H_{v}\left(Q, k_{x}, k_{y}, \omega\right)\right|^{2} \mathrm{~d} k_{x} \mathrm{~d} k_{y}
$$

where the transfer function $H_{v}\left(Q, k_{x}, k_{y}, \omega\right)$ is the structural response at point Q excited by a wall pressure plane wave of unit amplitude.

By discretizing this integral and introducing the ASD of the wave amplitudes [i.e. Eq. (7)], one obtains: : 


$$
S_{v v}(Q, \omega)=\sum_{r, s} S_{A_{r s} A_{r s}}(\omega)\left|H_{v}\left(Q, k_{r}, k_{s}, \omega\right)\right|^{2}
$$

This demonstrates that the structural velocity ASD function can be obtained from the effect of uncorrelated wall pressure plane waves simulating the TBL wall pressure fluctuations.

In theory, the numerical evaluation of the series given by Eq. (9) requires an infinite number of wavenumber couples $\left(k_{r}, k_{s}\right)$. However, the structure acts as a wave-vector filter characterized by the transfer function $H_{v}$. This enables limiting the wavenumber couples to those mainly contributing to the structural response $[25,28,29]$. Consequently, the structural velocity ASD function can be approximated from a finite number of uncorrelated wall pressure plane waves. This, however, requires some knowledge about the structure under test to determine an optimal cut-off wavenumber allowing properly truncating the series given by Eq. (9). Its practical evaluation is detailed in section 5 .

Concretely, the experimental process is divided in two main steps. In the first step the response $H_{v}\left(Q, k_{r}, k_{s}, \omega\right)$ of the structure excited by a wall pressure plane wave of unit amplitude is measured. This measurement is then repeated for a set of wall pressure plane waves with different wavenumbers. In the second step, these measurements are post-processed from Eq. (9). 


\section{Generation of wall pressure plane waves by a monopole array}

In section 2 , the concept of wall pressure plane wave is introduced for 2-dimensional plane waves propagating over a wall. In the following, wall pressure plane waves and acoustic plane waves must be distinguished, since the latter are used for denoting 3-dimensional plane waves.

\subsection{Basic principle}

In this section, the generation of a wall pressure plane wave of unit amplitude $\bar{P}_{r s}$ is investigated.

To do this, let us first consider an harmonic acoustic plane wave $p_{a c}$ of unit amplitude, propagating in an acoustic medium with the general form:

$$
p_{a c}(x, y, z, t)=e^{j \omega t+j k_{r} x+j k_{s} y+j k_{z} z}
$$

where $\omega$ is the angular frequency, $t$ is the time and $k_{z}$ is the wavenumber along the direction perpendicular to the $(x, y)$ plane in a Cartesian coordinate system.

The triplet $\left(k_{r}, k_{s}, k_{z}\right)$ is related to the acoustic wavenumber $k_{0}$ by the dispersion relation of the Helmholtz equation:

$$
k_{0}^{2}=k_{r}^{2}+k_{s}^{2}+k_{z}^{2}
$$

Insofar as the wavenumbers $k_{r}$ and $k_{s}$ are known, since chosen to suitably approximate the series given by Eqs. (4) and (9), the wavenumber $k_{z}$ satisfies: 


$$
\begin{cases}k_{z}= \pm \sqrt{k_{0}^{2}-k_{r}^{2}-k_{s}^{2}} & \text { if } k_{0}^{2}>k_{r}^{2}+k_{s}^{2} \\ k_{z}= \pm j \sqrt{k_{0}^{2}-k_{r}^{2}-k_{s}^{2}} & \text { if } k_{0}^{2}<k_{r}^{2}+k_{s}^{2}\end{cases}
$$

Depending on the value of $k_{r}^{2}+k_{s}^{2}$ with respect to $k_{0}^{2}$, Eq. (12) clearly indicates that wall pressure fields corresponding to those of propagating $\left(k_{z} \in \mathbb{R}\right)$ or evanescent $\left(k_{z} \in \mathbb{I}\right)$ acoustic plane waves must be generated to simulate TBL excitation from uncorrelated wall pressure plane waves. This is illustrated by Fig. (1) which compares the CSD functions associated with a diffuse field with a TBL wall pressure field.

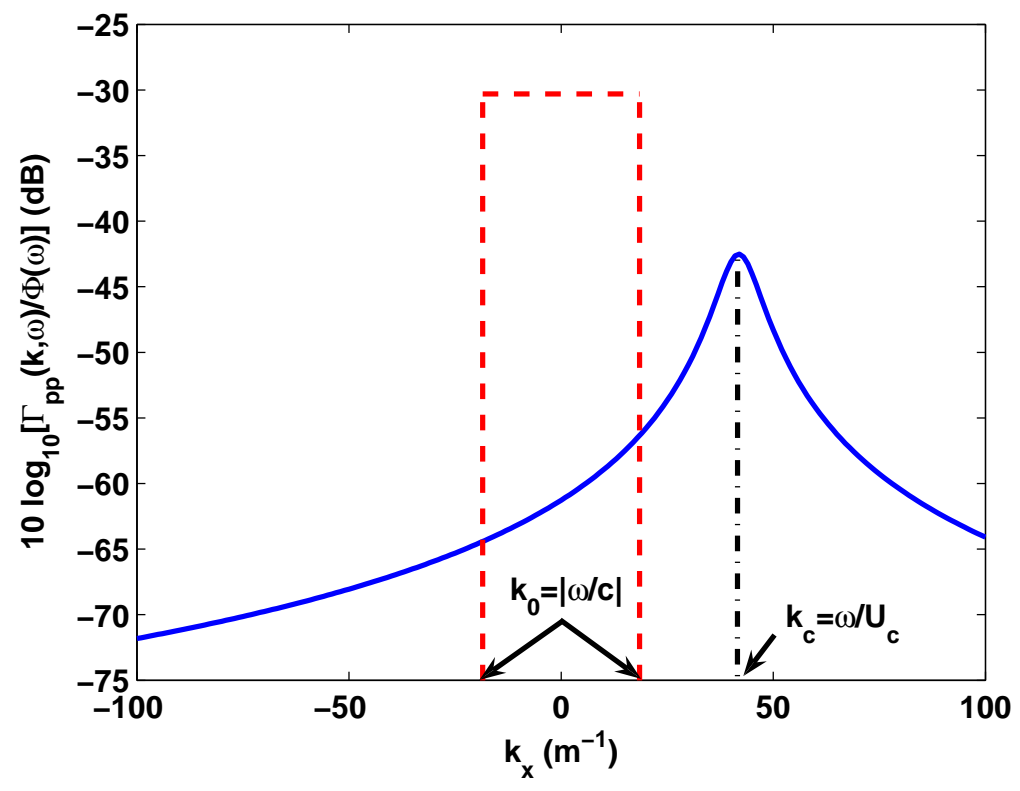

Figure 1: Comparison of cross-spectral density function of $(-)$ a TBL and (--) a diffuse field $-k_{c}$ : convective wavemenumber, $k_{0}$ : acoustic wavenumber

However, experimentally, generating evanescent acoustic plane waves nor- 
mal to the wall is very difficult. That is why, an array of acoustic monopoles is used instead to simulate the required nearfield interferential conditions. A schematic representation of such a device is proposed in Fig. 2.

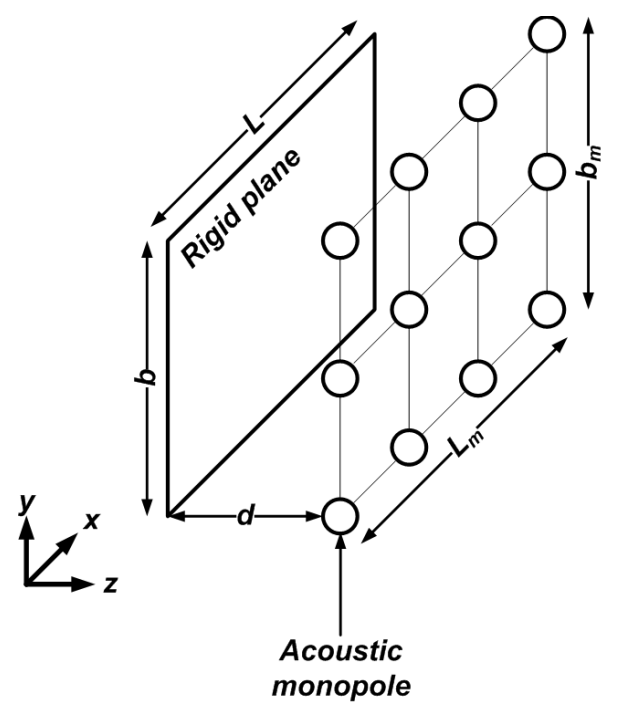

Figure 2: Definition of the array of acoustic monopoles

For each couple $\left(k_{r}, k_{s}\right)$, the complex amplitude $B_{m}^{r s}(\omega)$ of the m monopoles are obtained by writing the equality of a wall pressure plane wave of unit amplitude $\bar{P}_{r s}$ and the wall pressure field generated by the monopole array over a grid of p observation points located on a rigid plane:

$$
\sum_{m} B_{m}^{r s}(\omega) H_{m p}(\omega)=\mathrm{e}^{j k_{r} x_{p}+j k_{s} y_{p}}
$$

where $H_{m p}(\omega)$ is the transfer function between a monopole $m$ of coordinates $\left(x_{m}, y_{m}, z_{m}\right)$ and an observation point $\mathrm{p}$ of coordinates $\left(x_{p}, y_{p}, 0\right)$ belonging to a rigid wall. 
Considering an image source in order to satisfy the rigid wall condition, one classically obtains:

$$
H_{m p}(\omega)=\frac{\mathrm{e}^{-j k_{0} \sqrt{\left(x_{m}-x_{p}\right)^{2}+\left(y_{m}-y_{p}\right)^{2}+z_{m}^{2}}}}{2 \pi \sqrt{\left(x_{m}-x_{p}\right)^{2}+\left(y_{m}-y_{p}\right)^{2}+z_{m}^{2}}}
$$

For the sake of simplicity, Eq. (13) is written in matrix form:

$$
\mathbf{H B}=\mathbf{P}
$$

where $\mathbf{H}$ is the propagation operator corresponding to $H_{m p}$, B the vector of monopoles amplitude $B_{m}^{r s}$ and $\mathbf{P}$ the vector of the target wall pressure field corresponding to a wall pressure plane wave of unit amplitude $\bar{P}_{r s}$.

In most cases, the number of observation points $\mathrm{p}$ is greater than that of the monopoles $\mathrm{m}$. Therefore the propagation operator $\mathbf{H}$ is rectangular and Eq. (15) is solved in the least squares sense.

\subsection{Influence of the design parameters of the monopole array}

Three main parameters are involved in the design of the monopole array, namely the number of monopoles, its distance from the observation area and the size of the array. In this section, the influence of these parameters is studied numerically in order to determine the design rules used to describe the wall pressure plane waves.

To analyze the influence of each parameter separately, two types of results are studied. The first one consists in reconstructing a wall pressure plane wave reconstructed from the proposed approach [cf. Eq. (13)]. The second 
one is more global and concerns the reconstruction of the TBL CSD function from Eq. (4). In this study, theses reconstructions are performed at $250 \mathrm{~Hz}$ on a rectangular surface of $0.6 \times 0.3 \mathrm{~m}^{2}$. The TBL wall pressure fluctuations are described by the Corcos model [17] whose parameters are given in table 1:

$$
\Gamma_{p p}\left(k_{r}, k_{s}, \omega\right)=\frac{\Phi(\omega)}{k_{c}^{2}} \frac{4 \alpha_{x} \alpha_{y}}{\left[\alpha_{x}^{2}+\left(1-\frac{k_{r}}{k_{c}}\right)^{2}\right]\left[\alpha_{y}^{2}+\left(\frac{k_{s}}{k_{c}}\right)\right]^{2}}
$$

where $\Phi(\omega)$ is TBL wall pressure frequency spectrum, the constants $\alpha_{x}$ and $\alpha_{y}$ the longitudinal and lateral decay rates of the correlation and $k_{c}=\frac{\omega}{U_{c}}$ the convective wavenumber $\left(U_{c}\right.$ : convection velocity).

Table 1: Simulation parameters

\begin{tabular}{ll}
\hline Parameters & Values \\
\hline Free stream velocity & $U_{\infty}=50 \mathrm{~m} \cdot \mathrm{s}^{-1}$ \\
Convection velocity & $U_{c}=35 \mathrm{~m} \cdot \mathrm{s}^{-1}$ \\
Longitudinal decay rate & $\alpha_{x}=0.116$ \\
Lateral decay rate & $\alpha_{y}=0.7$ \\
TBL wall pressure spectrum at $250 \mathrm{~Hz}$ & $\Phi(\omega)=74 \mathrm{~Pa}^{2} \cdot{\mathrm{s} \cdot \mathrm{rad}^{-1}}$ \\
\hline
\end{tabular}

To reconstruct the TBL CSD function from a monopole array, a set of wall pressure plane waves is defined such that $\left(k_{r}, k_{s}\right) \in[-\bar{k}, \bar{k}]^{2}$, where $\bar{k}$ is a cut-off wavenumber. This particular wavenumber is defined to suitably truncate the series given by Eqs. (4) and (9) by taking into account the filtering effect of the structure on the TBL excitation. The practical choice of the cut-off wavenumber $\bar{k}$ is explained in section 5 . 
In the following simulations, $\bar{k}=50 \mathrm{~m}^{-1}$ and the wavenumber resolution is such that $\Delta k_{r}=\Delta k_{s}=1 \mathrm{~m}^{-1}$. This implies that the smallest wavelength to be reconstructed is $\bar{\lambda}=0.126 \mathrm{~m}$ leading to the definition of about $10^{4}$ wall pressure plane waves.

For the sake of clarity, we briefly explain the procedure used to obtain the wall pressure field of a wall pressure plane wave and then the CSD function of wall pressure fluctuations.

In a first step, in order to generate virtually a target wall pressure plane wave, the process consists in calculating the amplitude $B_{m}^{r s}$ of each monopole obtained from Eq. (13) for a grid of $50 \times 50$ observation points. Satisfying this equation allows reconstructing the wall pressure plane wave from the monopole array. This process is repeated for all the wall pressure plane waves simulating the TBL wall pressure fluctuations. To assess the robustness of our results, the wall pressure plane waves have to be reconstructed on a grid different from that used to calculate the amplitude $B_{m}^{r s}$ in order to assess the validity of the approach. Here, the wall pressure plane waves are reconstructed on a grid of $200 \times 200$ reconstruction points. The CSD function of the wall pressure fluctuations in the physical space, $S_{p p}\left(\xi_{x}, \xi_{y}, \omega\right)$ is estimated in a second step by summing the CSD function of the reconstructed wall pressure plane waves [supposed uncorrelated, see Eq(4)]. The reconstructed CSD function of the TBL wall pressure fluctuations in the wavenumber space, $\Gamma_{p p}\left(k_{x}, k_{y}, \omega\right)$, is deduced by using a FFT algorithm, whose parameters are summarized in table 2. 
Table 2: FFT parameters

\begin{tabular}{ll}
\hline Parameters & Values \\
\hline Length of the observation area & $L_{x}=0.6 \mathrm{~m}$ \\
Width of the observation area & $L_{y}=0.3 \mathrm{~m}$ \\
Spatial resolution & $(\Delta x, \Delta y)=(0.003 \mathrm{~m}, 0.0015 \mathrm{~m})$ \\
FFT resolution & $M=2048$ and $N=4096$ \\
Wavenumber range of $k_{x}$ & {$\left[-\frac{\pi}{\Delta x}, \frac{\pi}{\Delta x}\right]\left(\frac{\pi}{\Delta x}=1047 \mathrm{~m}^{-1}\right)$} \\
Wavenumber range of $k_{y}$ & {$\left[-\frac{\pi}{\Delta y}, \frac{\pi}{\Delta y}\right]\left(\frac{\pi}{\Delta y}=2094 \mathrm{~m}^{-1}\right)$} \\
Wavenumber resolution & $\left(\Delta k_{x}, \Delta k_{y}\right)=\left(\frac{2 \pi}{M \Delta x}, \frac{2 \pi}{N \Delta y}\right) \approx\left(1 \mathrm{~m}^{-1}, 1 \mathrm{~m}^{-1}\right)$ \\
\hline
\end{tabular}

In the following sections, two types of figures are presented, namely a wall pressure plane wave for $\left(k_{r}, k_{s}\right)=(\bar{k}, \bar{k})$ and the CSD functions of wall pressure fluctuations. The first type of figures highlights the quality of reconstruction of a wall pressure plane wave. The second type of figures indicates how the reconstruction of wall pressure fluctuations is correct when the contributions of all the wall pressure plane waves are taken into account.

\subsubsection{Optimal number of acoustic monopoles}

To determine the optimal number of acoustic monopoles, it is first assumed that the observation area and the array have the same size and the distance between them is $\frac{\bar{\lambda}}{2}=0.063 \mathrm{~m}$, i.e. half the smallest wavelength to be reconstructed.

Figs. 3 and 4 illustrate the influence of the number of monopoles on the reconstructions of the TBL CSD function and a wall pressure plane wave for 
$\left(k_{r}, k_{s}\right)=(\bar{k}, \bar{k})$, when using $2,3,4$ and 6 monopoles per wavelength $\bar{\lambda}$.

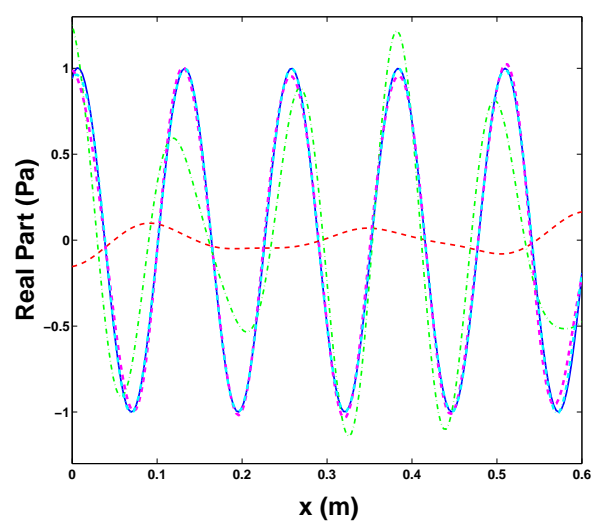

(a)

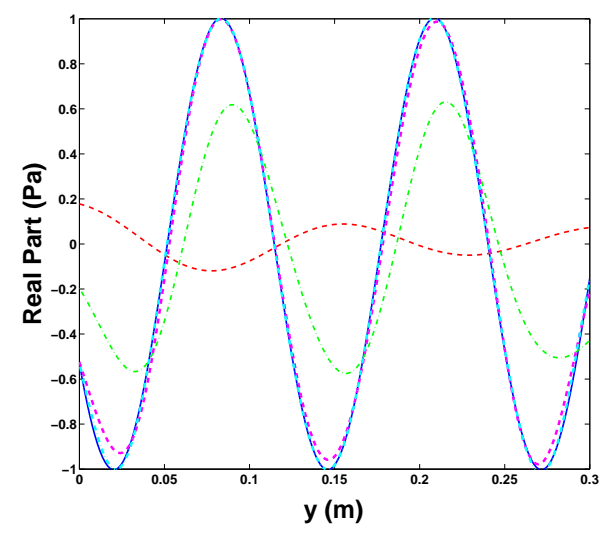

(b)

Figure 3: Influence of the number of acoustic monopoles on the reconstruction of a wall pressure plane wave for $\left(k_{r}, k_{s}\right)=(\bar{k}, \bar{k})$ at $250 \mathrm{~Hz}$ in planes (a) $(x, 0.12 \mathrm{~m})$ and (b) (0.3 $\mathrm{m}, y)-(-)$. Reference, (--) 2 monopoles per wavelength $\bar{\lambda},(-,-), 3$ monopoles per wavelength $\bar{\lambda},(--), 4$ monopoles per minimal $\bar{\lambda}$ and (-॰-), 6 monopoles per wavelength $\bar{\lambda}$

Figs. 3 and 4 clearly show that a minimum of 4 monopoles per wavelength $\bar{\lambda}$ is required to reconstruct the TBL CSD function and the wall pressure plane wave correctly, since using 2 or 3 monopoles per wavelength leads to poor reconstructions. For the real-time synthesis of TBL pressure fields, Maury and Bravo $[14,15]$ proposed an empirical criterion for an accurate reconstruction from an array of loudspeakers. This criterion gives respectively 3.7 per unit of spanwise correlation length in the streamwise direction and 3 sources per unit of spanwise correlation length in the spanwise direction. However, even if these criteria seem consistent together, they cannot be directly compared since, in the present case, our empirical criterion is based 


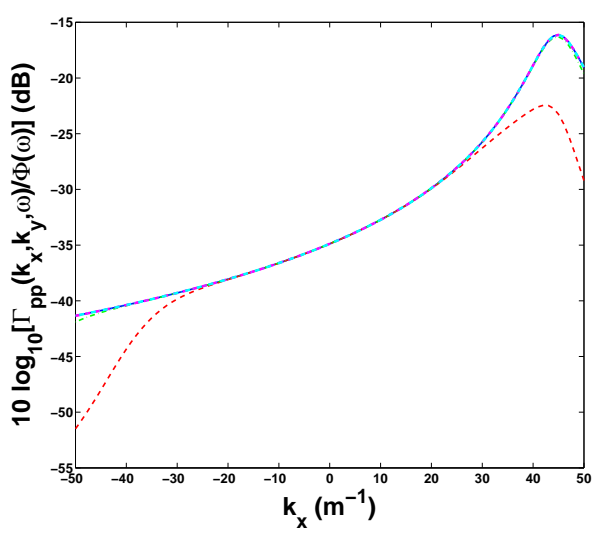

(a)

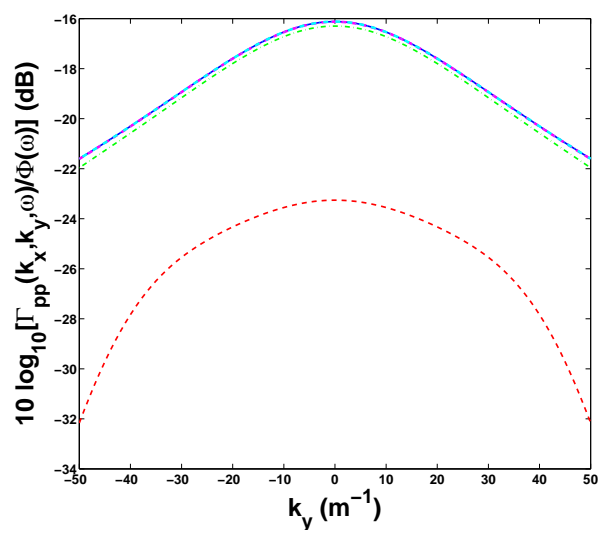

(b)

Figure 4: Influence of the number of acoustic monopoles on the reconstruction of the TBL cross-spectral density function given by the Corcos model in planes (a) $\left(k_{x}, 0\right)$ and (b) $\left(k_{c}, k_{y}\right)$ at $250 \mathrm{~Hz}-(-)$. Reference, $(--) 2$ monopoles per wavelength $\bar{\lambda},(-.-), 3$ monopoles per wavelength $\bar{\lambda},(--), 4$ monopoles per minimal $\bar{\lambda}$ and (-.-), 6 monopoles per wavelength $\bar{\lambda}$ 
on the wavelength to reconstruct and not on the spanwise correlation length. Nevertheless, both criteria predict an increase in the number of monopoles with frequency. Here, this is due to the increase of cut-off wavenumber $\bar{k}$ with frequency in order to take into account the filtering effect of the structure on TBL excitation. Consequently, this can lead to substantial monopole density at high frequencies, which would be unrealistic to implement experimentally.

\subsubsection{Distance of the array from the observation area}

In Refs. [11, 14], it is shown for an array of loudspeakers modelled as an array of acoustic monopoles, that reconstructions are relatively independent of the distance between the array and the observation plane provided it is greater than the loudspeakers separation distance. Here, the idea is to specify the validity bounds of this parameter.

In order to study this parameter, the array is made up of 4 monopoles per wavelength $\bar{\lambda}$ and its size is assumed to be equal to that of the observation area. Figs. 5 and 6 present the influence of distance $d$ of the array from the observation area on the reconstructions of the TBL CSD function and the wall pressure plane wave, when distance $d$ is defined in the interval $\left[\frac{\bar{\lambda}}{6}, 4 \bar{\lambda}\right]$.

As expected, Figs. 5 and 6 show that reconstructions are relatively independent of the distance from the observation area. However, in Fig. 5 it can be seen that the quality of reconstruction of the wall pressure plane wave is poor when the array and the observation area are very close together, i.e. for $d=\frac{\bar{\lambda}}{6}$. This can be explained by the poor acoustic coverage of the observation area, since an observation point is mainly influenced by the closer 


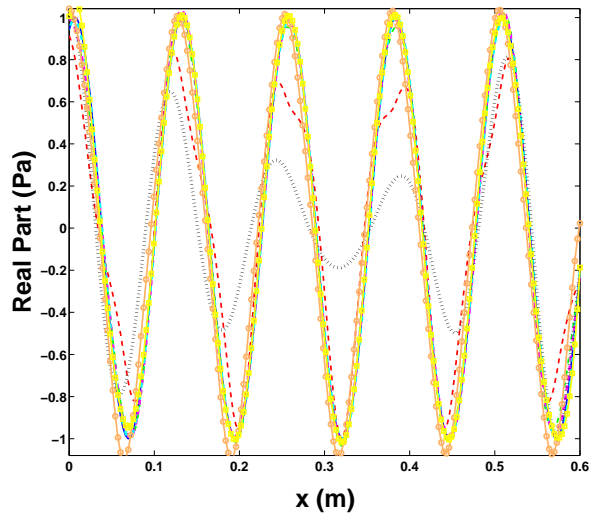

(a)

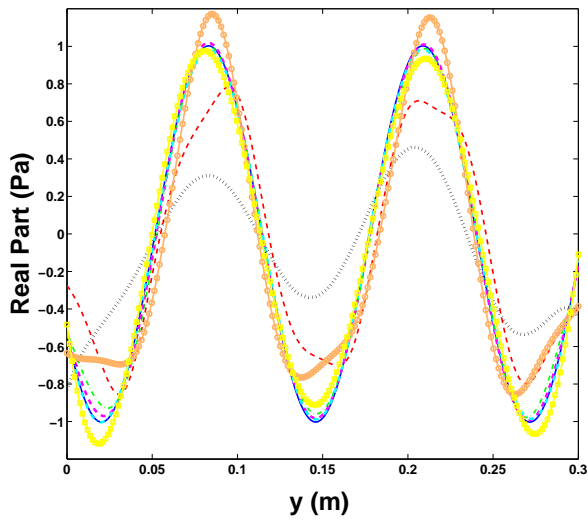

(b)

Figure 5: Influence of the distance of the array to the observation area on the reconstruction of a wall pressure plane wave for $\left(k_{r}, k_{s}\right)=(\bar{k}, \bar{k})$ at $250 \mathrm{~Hz}$ in the planes (a) $(x, 0.12$ $\mathrm{m})$ and (b) $(0.3 \mathrm{~m}, y)-(-)$ Reference, $(--) d=\frac{\bar{\lambda}}{6},(-\mathrm{o}-) d=\frac{\bar{\lambda}}{4},(-,-) d=\frac{\bar{\lambda}}{2},(--)$ $d=\bar{\lambda},(-\square-) d=3 \bar{\lambda}$ et $(\ldots) d=4 \bar{\lambda}$ 


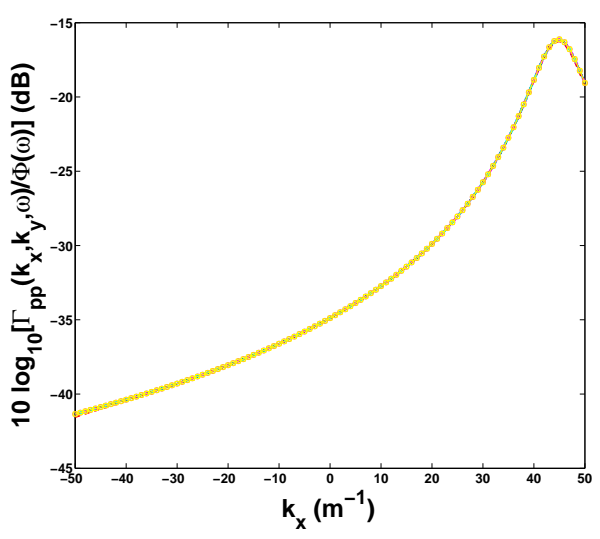

(a)

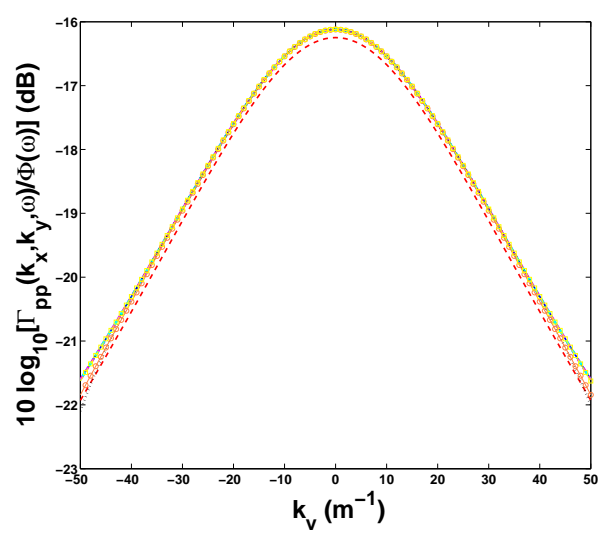

(b)

Figure 6: Influence of the distance of the array to the observation area on the reconstruction of the TBL cross-spectral density function given by the Corcos model in the planes (a) $\left(k_{x}, 0\right)$ and (b) $\left(k_{c}, k_{y}\right)$ at $250 \mathrm{~Hz}-(-)$ Reference, $(--) d=\frac{\bar{\lambda}}{6},(-\mathrm{O}-) d=\frac{\bar{\lambda}}{4},(-,-)$ $d=\frac{\bar{\lambda}}{2},(--) d=\bar{\lambda},(-\square-) d=3 \bar{\lambda}$ et $(\ldots) d=4 \bar{\lambda}$ 
monopole. Likewise, the further the array is from the observation area, the less two neighbouring monopoles can be differentiated, causing the propagating operator $\mathbf{H}$ to be ill-conditioned. Fig. 5 shows that this situation occurs for $d=4 \bar{\lambda}$. Consequently, to avoid these extreme cases, the distance between the array and the observation area has to be set in the interval $[\bar{\lambda}, 3 \bar{\lambda}]$.

\subsubsection{Size of the monopole array}

In this section, we verify the sensitivity of the size of the monopole array on the reconstructions of the TBL CSD function and the wall pressure plane wave. Bravo and Maury [14] have addressed this issue by determining the maximal synthesis frequency as a function of the number of loudspeakers, the aperture of the array and the distance of the array to the observation area. A different approach, adapted to our methodology, is proposed. To this end, the array is made up of 4 monopoles per wavelength $\bar{\lambda}$. The distance between the array and the observation area is still $\frac{\bar{\lambda}}{2}=0.063 \mathrm{~m}$.

Figs. 7 and 8 show the influence of the size of the array on the reconstructions of the TBL CSD function and a wall pressure plane wave, when the size of the array is defined as a fraction $d_{a}$ of the size of the observation area, varying between $\frac{1}{2}$ and 2 . In other words, length $L_{m}$ and width $b_{m}$ of the array are such that $L_{m}=d_{a} L$ and $b_{m}=d_{a} b$, with $d_{a}$ ( $L$ and $b$ : length and width of the observation area).

Figs. 7 and 8 show in particular that the size of the array has to be equal to or greater than the size of the observation area to reconstruct properly the shape of each wall pressure plane wave by ensuring a good acoustic coverage. 


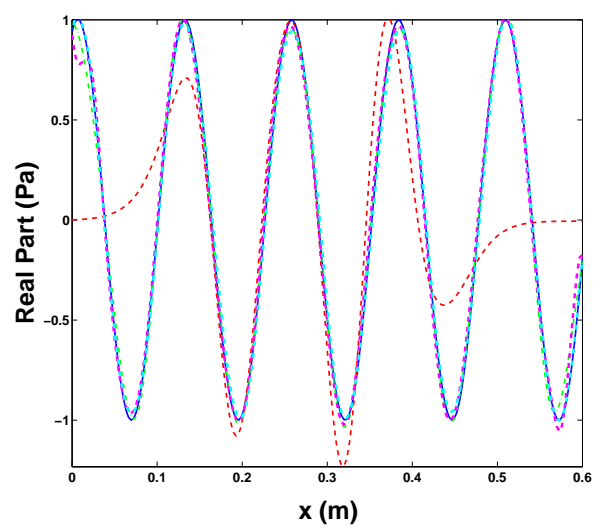

(a)

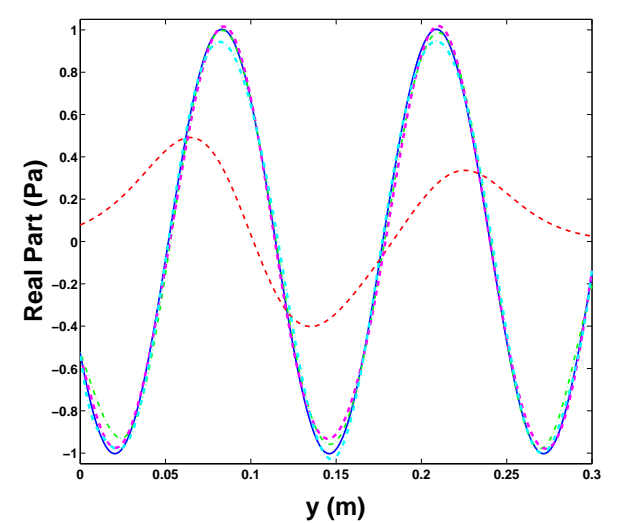

(b)

Figure 7: Influence of the size of the monopole array on the reconstruction of a wall pressure plane wave for $\left(k_{r}, k_{s}\right)=(\bar{k}, \bar{k})$ at $250 \mathrm{~Hz}$ in the plane (a) $(x, 0.12 \mathrm{~m})$ and (b) $(0.3 \mathrm{~m}, y)-(-)$ Reference, $(--) d_{a}=0.5,(-.-) d_{a}=1,(--) d_{a}=1.5,(-.-) d_{a}=2$

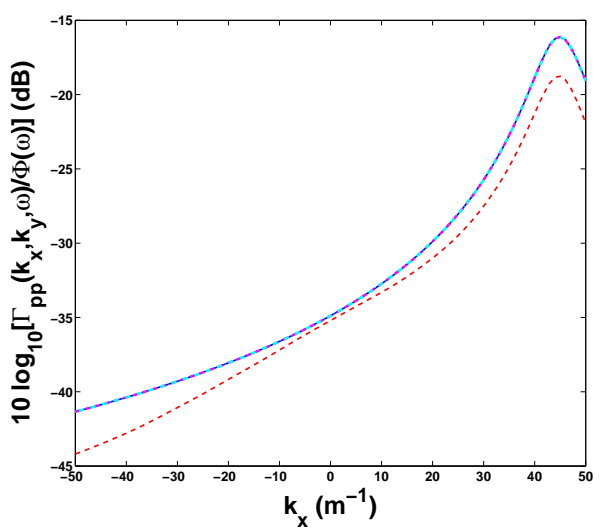

(a)

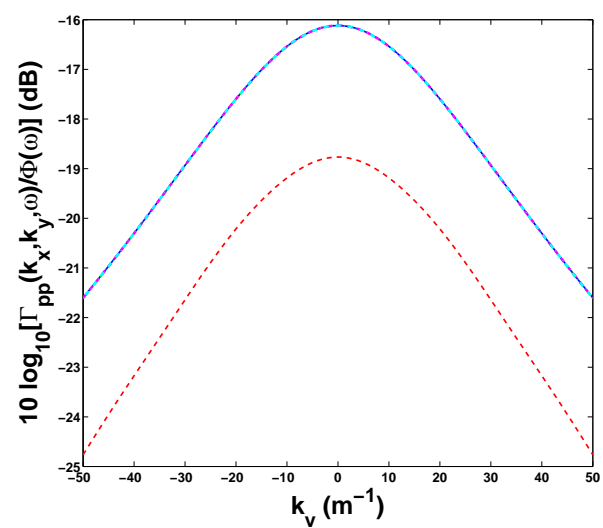

(b)

Figure 8: Influence of the size of the monopole array on the reconstruction of the TBL cross-spectral density function given by the Corcos model in the planes (a) $\left(k_{x}, 0\right)$ and (b) $\left(k_{c}, k_{y}\right)$ at $250 \mathrm{~Hz}-(-)$ Reference, $(--) d_{a}=0.5,(-,-) d_{a}=1,(--) d_{a}=1.5,(-.-)$ $d_{a}=2$ 
Indeed, when the size of the array is smaller than that of the observation area, the number of monopoles is too low to properly describe the spatial variations of the wall pressure plane wave. Finally, it is useful to note that the larger the array, the more the number of monopoles increases. In practice, this would mean using a monopole array of the same size as the observation area.

\subsubsection{Summary of the parametric study}

In the design of the monopole array, three main parameters intervene, that is the number of monopoles of the array, its distance from the observation area and its size. The previous parametric study allows concluding that (a) a minimum of 4 monopoles per wavelength $\bar{\lambda}$ is required to properly reconstruct a wall pressure plane wave (b) the distance between the array

and the observation area can be arbitrarily chosen in the interval $\left[\frac{\bar{\lambda}}{4}, 3 \bar{\lambda}\right]$ and (c) the size of the monopole array could be, at least, the same as the observation area.

This predesign parametric study has been performed by considering theoretical acoustic monopoles. In practice, monopole-like sources differ from theoretical sources, since the acoustic pressure field is not singular in the very nearfield of the source. However, it has been pointed out that the source array should not be too close to the observation area to avoid an ill-conditioning of the propagation operator $\mathbf{H}$. Consequently, one can reasonably expect that above results remain acceptable for real acoustic monopole-like sources, as will be shown in section 5 . 


\section{Using a synthetic array for experimentally simulating TBL in- duced vibrations of panels}

As highlighted in section 2.3, one of the keys to the proposed experimental procedure is the measurement of the transfer function $H_{v}\left(Q, k_{r}, k_{s}, \omega\right)$. Insofar as this function can be viewed as the response of the structure to a wall pressure plane wave of unit amplitude, we propose using, in section 3, a monopole array to generate such a pressure field. However, as shown in section 3.2.1, this can be a tricky task since the density of monopoles increases with frequency. Consequently, this limits the experimental simulation to the low frequency range, making it necessary to use a synthetic array to avoid frequency limitation due to sources density.

The principle of the synthetic array relies on the linearity of the problem. It was shown in section 3.1 that the superposition principle could be used for simulating a wall pressure plane wave from a monopole array. An immediate consequence is the possibility of simulating a wall pressure plane wave by combining the individual contribution of each monopole separately. The proposed approach is based on this result and consists in using a single moving monopole-like source to reconstruct a wall pressure plane wave from sequential measurements.

The experimental process, considered in this paper, is derived from high resolution synthetic aperture Sonar [30]. This technique consists in post processing the signals of a moving receiving array to reconstruct an unknown target image, or emitter, with a finer spatial resolution than that obtained with a fixed array. In other words, a large virtual array is simulated from a 
small physical one.

In this paper, the reciprocal mechanism is used, since the moving array is the emitter. To properly implement the synthetic array approach in the proposed methodology, the evaluation of the transfer function $H_{v}\left(Q, k_{r}, k_{s}, \omega\right)$ requires two measurements, the first one to characterize the acoustic medium and the second one to characterize the behaviour of the structure excited by an acoustic monopole. By combining both information, one can obtain an evaluation of the target transfer function. Consequently, the implementation of the synthetic array approach is carried out in 3 steps, which are detailed in this section.

\subsection{Step 1: Simulation of wall pressure plane waves by a synthetic array}

Step 1 concerns the simulation of a set of wall pressure plane waves of unit amplitude using the synthetic array. These wall pressure plane waves are input data to compute the amplitude $B_{m}^{r s}$ for each position of the monopole-like source and each couple of wavenumbers $\left(k_{r}, k_{s}\right)$ [see Eq. (13)]. Practically speaking, this calculation requires the knowledge of the transfer functions $H_{m p}$ between the particle velocity of the monopole-like source $\mathrm{m}$ and the pressure at observation points $\mathrm{p}$. In a real acoustic situation, these transfer functions do not correspond exactly to the half-space Green's function. Consequently, they have to be determined from sequential measurements of the blocked pressure on a rigid wall due to the monopole-like source, as shown in Fig. 9. 


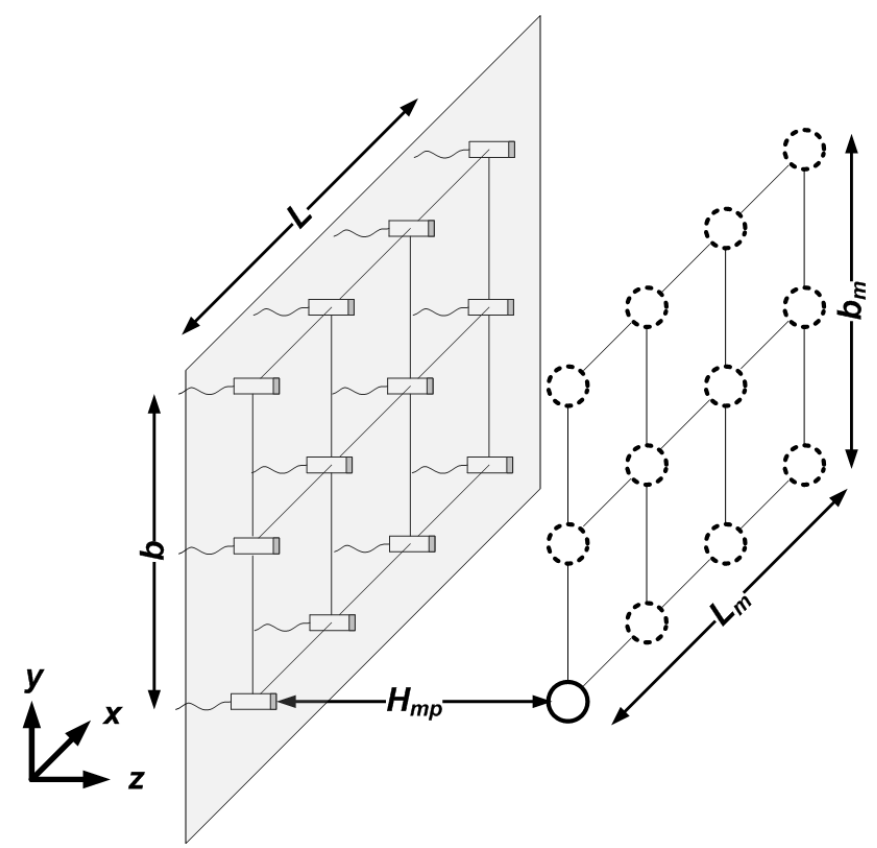

Figure 9: Example of measurement of the transfer functions $H_{m p}$

\subsection{Steps 2 and 3: Reconstruction of TBL induced panel vibrations}

To characterize the vibratory behaviour of the structure under consideration from step 1, it is first necessary to measure the transfer functions $H_{Q m}$ between the structural velocity at point $\mathrm{Q}$ and the particle velocity of the monopole-like source m. This procedure is illustrated in Fig. 10. Doing so, one obtains the response at point Q of the structure excited by an acoustic monopole of unit amplitude.

The calculation of TBL induced vibrations requires a numerical processing. The procedure first consists in multiplying the transfer function $H_{Q m}$ by amplitude $B_{m}^{r s}$, to obtain the contribution of one source to the transfer function $H_{v}\left(Q, k_{r}, k_{s}, \omega\right)$. The contributions for each position of the monopole-like 


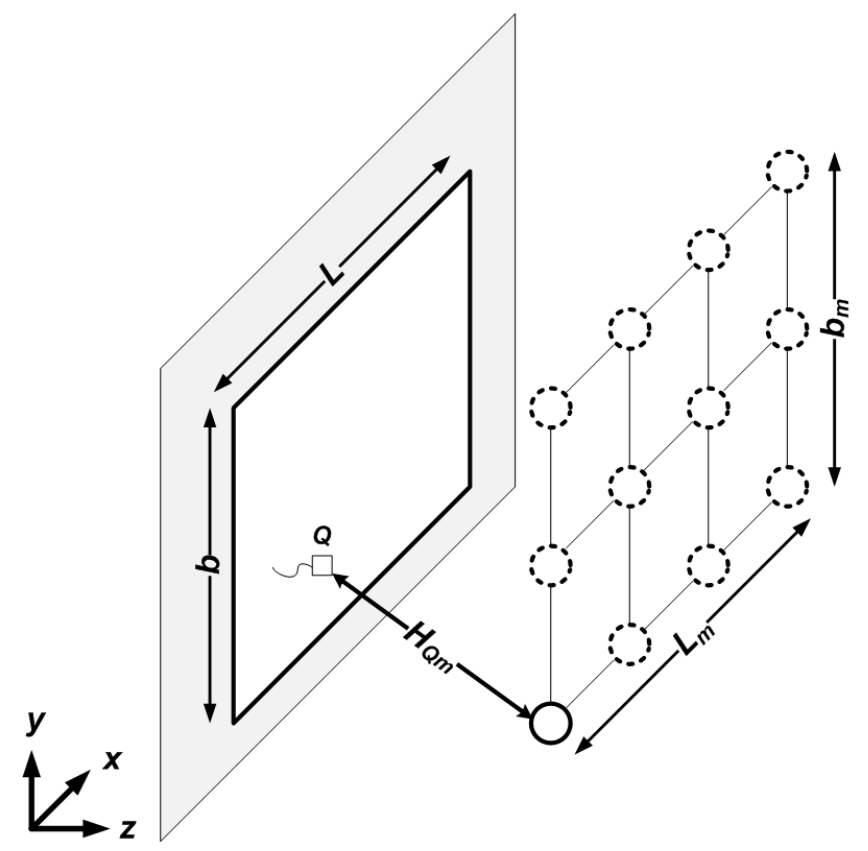

Figure 10: Example of measurement of the transfer functions $H_{Q m}$

source are then combined, by linear processing, to obtain the transfer function $H_{v}\left(Q, k_{r}, k_{s}, \omega\right)$ excited by a wall pressure plane wave of unit amplitude:

$$
H_{v}\left(Q, k_{r}, k_{s}, \omega\right)=\sum_{m} H_{Q m}(\omega) B_{m}^{r s}(\omega)
$$

The structural velocity ASD function is finally obtained by using Eq. (9).

\subsection{Summary of the experimental process}

In this section, we propose to summarize, under a block diagram form [cf. Fig. (11)], the experimental process detailed above in order to clearly distinguish measurement stages from numerical processing stages based on measured data. 


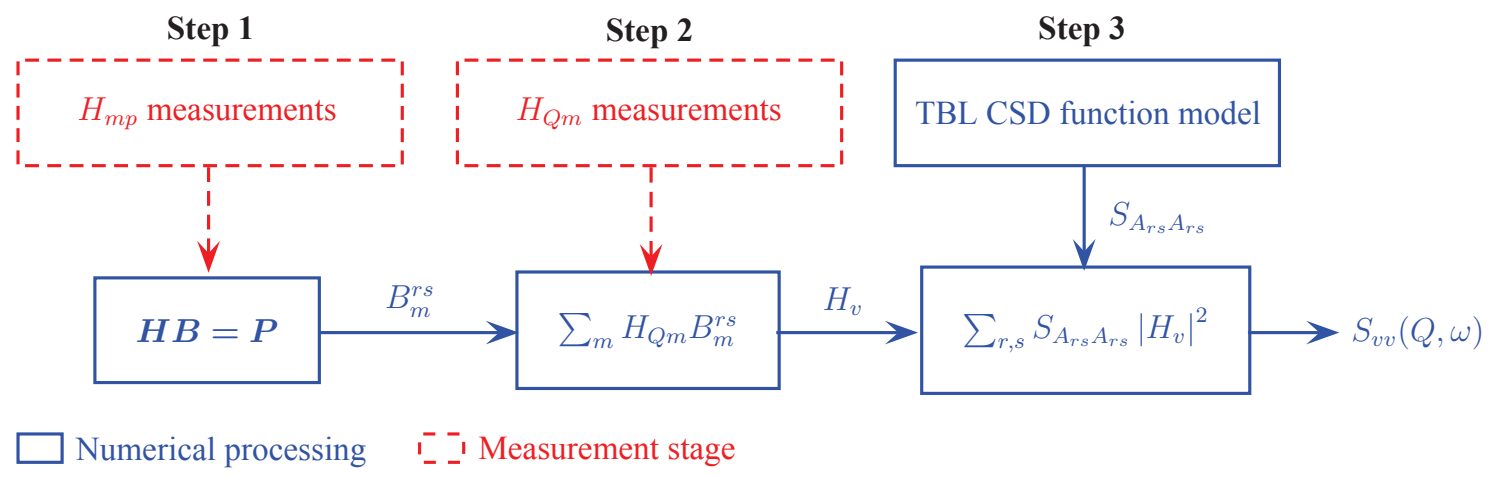

Figure 11: Block diagram of the proposed experimental procedure

The proposed block diagram shows that the use of a synthetic array requires two transfer functions measurements and numerical processings. Such a process presents several advantages to assess TBL induced vibrations. Firstly, a synthetic array allows greater flexibility with respect to the number of monopoles required for the simulation. Then, from the practical standpoint, only transfer function measurements are required. These two latter points are very interesting, since it is not necessary to control the amplitude and phase of several sources simultaneously. Furthermore, dispersions of sensor characteristics are avoided as are all the reference problems occurring when an array of sources is used. Nevertheless, the sequential nature of the experimental process requires measurements to be made with care. Indeed, inaccuracies of source positioning can generate phase shift dispersions between two successive positions of the source. This problem can be easily managed by using a two-axis robot as done in section 5. Another issue is related to the variations of the set-up with time, since sequential mea- 
surements are quite lengthy. Consequently, the behaviour of the structure may change during the experiment, because of environmental variations for instance. To insure the consistency of measurements throughout the experiment presented in the next section, the temperature and the hygrometry were monitored with a wireless weather station.

\section{Experimental validation on an academic structure}

In this section, an experimental validation of the synthetic array approach is proposed for a $0.6 \times 0.3 \times 0.0005 \mathrm{~m}^{3}$ steel plate for frequencies below 250 $\mathrm{Hz}$.

\subsection{Definition of experimental parameters}

As explained in section 2, the filtering effect of the structure with respect to the TBL excitation allows limiting the number of uncorrelated wall pressure plane waves necessary to calculate the velocity ASD function at point Q [see Eq. (9)] with good approximation. To this end, a cut-off wavenumber $\bar{k}$ was introduced in section 3 . A simple indicator for the practical choice of $\bar{k}$ can be derived from the analysis of the physical mechanisms governing the response of a plate excited by a TBL, such as the aerodynamic coincidence and the filtering effect of the structure (see Fig. 12).

For a plate with natural bending wavenumber $k_{f}$, immersed in a light fluid such as air, the aerodynamic coincidence associated with the filtering effect of the structure on the TBL excitation allows explaining the physical mechanisms of the TBL induced vibrations in a straightforward manner. In 

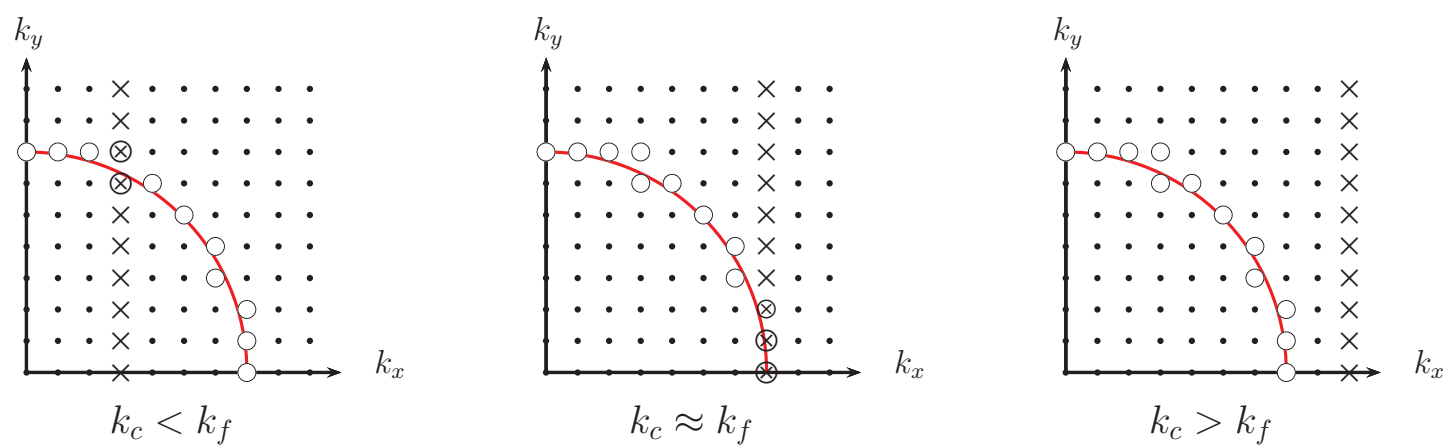

- Non-resonant modes

- Resonant modes

$\times$ Non-resonant modes in aerodynamic coincidence

$\otimes$ Resonant modes in aerodynamic coincidence

Figure 12: Principle of aerodynamic coincidence [10] $-k_{c}$ : convective wavenumber, $k_{f}$ : natural wavenumber of the structure

general, three configurations are observed. Indeed, for $k_{c}<k_{f}$, the resonant and non-resonant modes in aerodynamic coincidence of the plate are the main contributors to vibration response. For $k_{c} \approx k_{f}$, the number of resonant modes in aerodynamic coincidence is maximal [10]. In this case, these modes mainly contribute to the vibration response. Finally, for $k_{c}>k_{f}$, only the resonant modes have a significant contribution to the vibration response due to the filtering effect of the plate.

As a result, the wavenumbers $\left(k_{r}, k_{s}\right)$ defining the wall pressure plane waves can be restrained to those matching with the modes, that mainly contribute to the vibration response. Furthermore, this analysis implies a substantial reduction in the number of positions of the monopole-like source, since only the plane waves matching with the most important modes have to be re- 
constructed (see Fig. 12, after [10]). However, the a priori determination of structural modes as well as natural wavenumber $k_{f}$ can be a challenging task for industrial structures. For all these reasons, it is preferable to defined a simple but robust indicator to take into account all the physical mechanisms above-cited. Here, a practical choice of the cut-off wavenumber $\bar{k}$ is such that $\bar{k}>k_{f}$, where $k_{f}$ is the natural wavenumber of the plate equivalent to the real structure. Based on this indicator, the set of wall pressure plane waves is thus defined to satisfy $\left(k_{r}, k_{s}\right) \in[-\bar{k}, \bar{k}]^{2}$ with a prescribed wavenumber resolution $\left(\Delta k_{r}, \Delta k_{s}\right)$. Of course, to take advantage of the filtering effect of the structure on the excitation, it is necessary to have a good knowledge of the structure under test. In particular, this means that for industrial structures, such as multilayer or ribbed structures, the dynamic behaviour has to be studied carefully to properly identify the cut-off wavenumber.

In the experiment described here, the cut-off wavenumber $\bar{k}$ is set at $45 \mathrm{~m}^{-1}$, which is slightly higher than the value of the bending wavenumber $k_{f}$ of the plate at $250 \mathrm{~Hz}$, namely, $44.7 \mathrm{~m}^{-1}$. Then, the set of wall pressure plane waves is defined such that $\left(k_{r}, k_{s}\right) \in[-\bar{k}, \bar{k}]^{2}$ with a wavenumber resolution of $\Delta k_{r}=\Delta k_{s}=1 \mathrm{~m}^{-1}$, which approximately represents a set of 8300 wall pressure plane waves.

According to the results obtained in section 3, it is first assumed that the observation area and the virtual monopole array have the same size while the distance between them is $15 \mathrm{~cm}$, which corresponds approximately to the minimal wavelength $\bar{\lambda}$ to be reconstructed, namely $13.9 \mathrm{~cm}$. Then, ac- 
cording to the spatial characteristics of the wall pressure plane waves to be reconstructed, implementing the synthetic array approach requires 128 positions of the source, i.e. 16 along the length and 8 along the width of the virtual monopole array. These source positions are defined on the basis of 4 monopoles per minimal wavelength $\bar{\lambda}$. Finally, a grid of $21 \times 11$ observation points was used to calculate the amplitude $B_{m}^{r s}$ for each source location.

For the sake of clarity the experimental parameters are summarized in table 3 .

Table 3: Experiment parameters

\begin{tabular}{ll}
\hline Parameters & Values \\
\hline Size of the observation area & $L_{p} \times b_{p}=0.6 \times 0.3 \mathrm{~m}^{2}$ \\
Size of the virtual monopole array & $L_{m} \times b_{m}=0.6 \times 0.3 \mathrm{~m}^{2}$ \\
Number of positions of the source & $128(16 \times 8)$ \\
Number of observation points & $231(21 \times 11)$ \\
Distance monopole-observation area & $d=15 \mathrm{~cm}$ \\
Cut-off wavenumber & $\bar{k}=45 \mathrm{~m}^{-1}$ \\
Wavenumber resolution & $\Delta k_{r}=\Delta k_{s}=1 \mathrm{~m}^{-1}$ \\
Number of wall pressure plane waves & 8281 \\
Maximal frequency studied & $f_{\max }=250 \mathrm{~Hz}$ \\
\hline
\end{tabular}

\subsection{Experimental set-up}

\subsubsection{Description of the monopole-like source}

The experimental implementation of the synthetic array approach requires using an acoustic monopole as a source. However, since the concept 
of acoustic monopole is only theoretical, the first step consists in using an acoustic source whose properties are similar to those of an acoustic monopole in the frequency range of interest.

In this study, the monopole-like source is generated physically by a loudspeaker that emits white noise through a tube whose diameter is smaller than a third of the acoustic wavelength (cf. Fig. 13). The reference signal is measured by a velocity sensor fitted in a small nozzle mounted at the end of the tube, in order to take into account the standing waves existing in the tube. The inner diameter of the nozzle is $15 \mathrm{~mm}$. Consequently, the upper frequency for a monopole-like behaviour of the source is much lower than 7.5 $\mathrm{kHz}$. Furthermore, the lower frequency of the source is related to the signalto-noise ratio (SNR), as the monopole source is not an efficient radiator at low frequency. Preliminary measurements showed that SNR was acceptable for frequencies above $70 \mathrm{~Hz}$. Consequently, the experimental results are presented from $70 \mathrm{~Hz}$ to $250 \mathrm{~Hz}$.

\subsubsection{Experimental set-up for $H_{m p}$ measurements}

The measurements of the transfer function $H_{m p}$ between the particle velocity of the monopole-like source $\mathrm{m}$ and the pressure at the observation points $\mathrm{p}$ were carried out in a sound-treated room by using white-noise excitation and measuring the blocked pressure on a rigid wall, consisting of a thick wooden plate $1.02 \times 1.25 \times 0.036 \mathrm{~m}^{3}$, as presented in Fig. 14 . To this end, a two-axis robot was used to move the monopole-like source on the 128-positions defined previously and a nearfield linear microphone array with 21 1/4"-sensors spaced $3 \mathrm{~cm}$ apart was used to measure the blocked pressure 


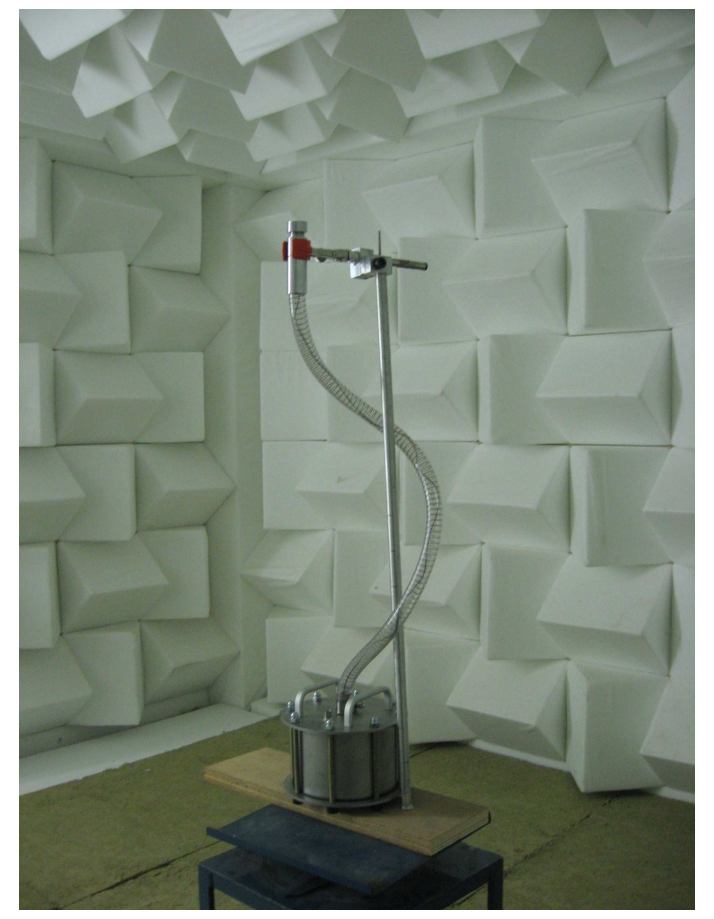

Figure 13: Monopole-like source 
over a grid of 231 observation points.

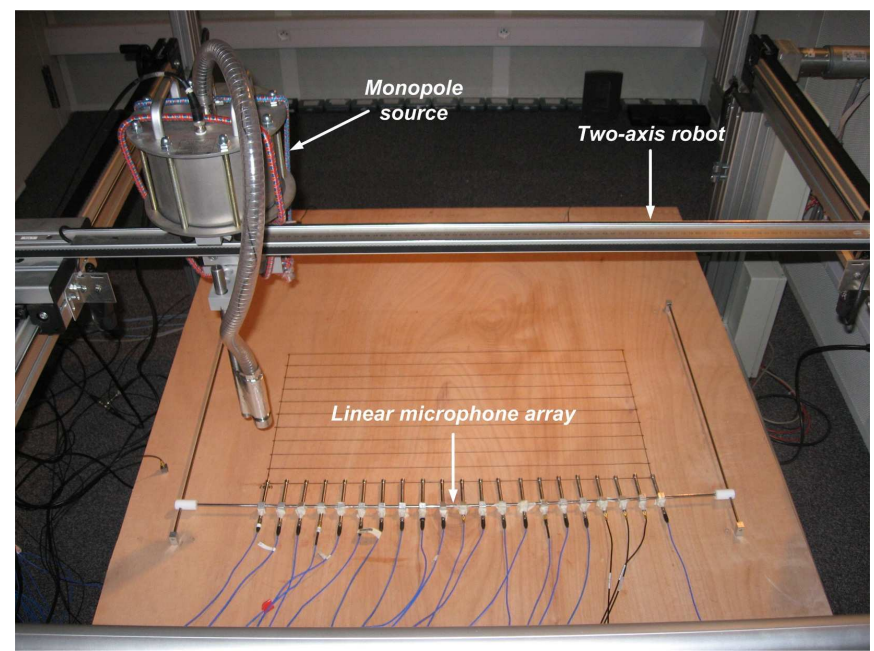

Figure 14: Experimental set-up for measuring the transfer functions $H_{m p}$

Fig. 15 presents a typical measurement of the transfer function $H_{m p}$ between one position of the monopole-like source and one nearfield microphone. It can be seen that the general trend of the transfer function increases with frequency, demonstrating better radiation efficiency of the monopolelike source with frequency.

Finally, it can be notice that measuring all the transfer functions $H_{m p}$ takes about 5 and a half hours for the experimental parameters given in table 3 . 


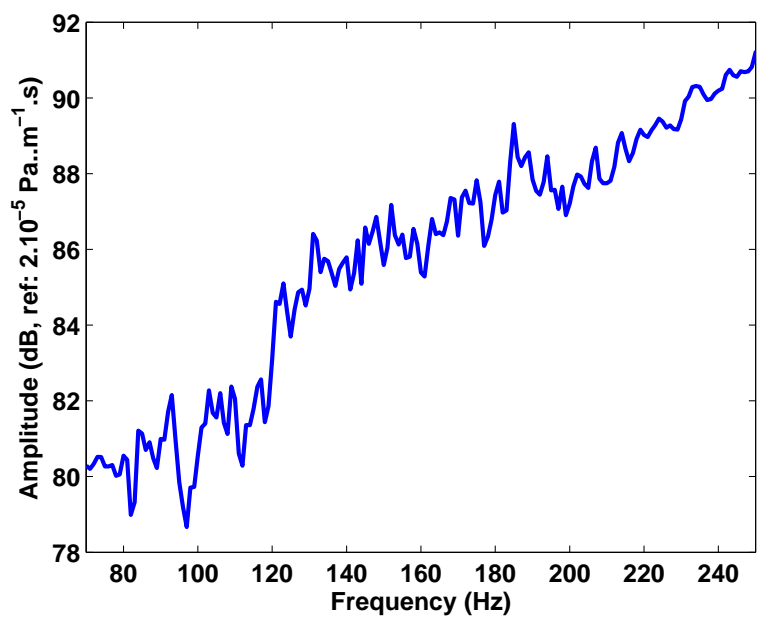

Figure 15: Typical result of $H_{m p}$ measurements - Modulus of the transfer function $\left|H_{m p}\right|$ measured between the particle velocity of the monopole-like source and the blocked pressure induced on a thick wooden plate with dimensions $1.02 \times 1.25 \times 0.036 \mathrm{~m}^{3}$

\subsubsection{Experimental set-up for $H_{Q m}$ measurements}

In this section, the measurements of the transfer functions $H_{Q m}$ are presented. As shown in Fig. 16, an aperture of dimensions $0.6 \times 0.3 \mathrm{~m}^{2}$ was made in the thick wooden plate used for $H_{m p}$ measurements, above which the plate studied was glued to allow bending motions. The measurements consisted in measuring the transfer function between the particle velocity of the monopole-like source and the structural velocity at an observation point Q located on the plate. To do this, white-noise excitation was used and structural velocity measurements were performed with a lightweight 4 gram accelerometer to limit the effect of the added mass of the sensor on the plate.

A typical measurement of the transfer functions $H_{Q m}$ is presented in 


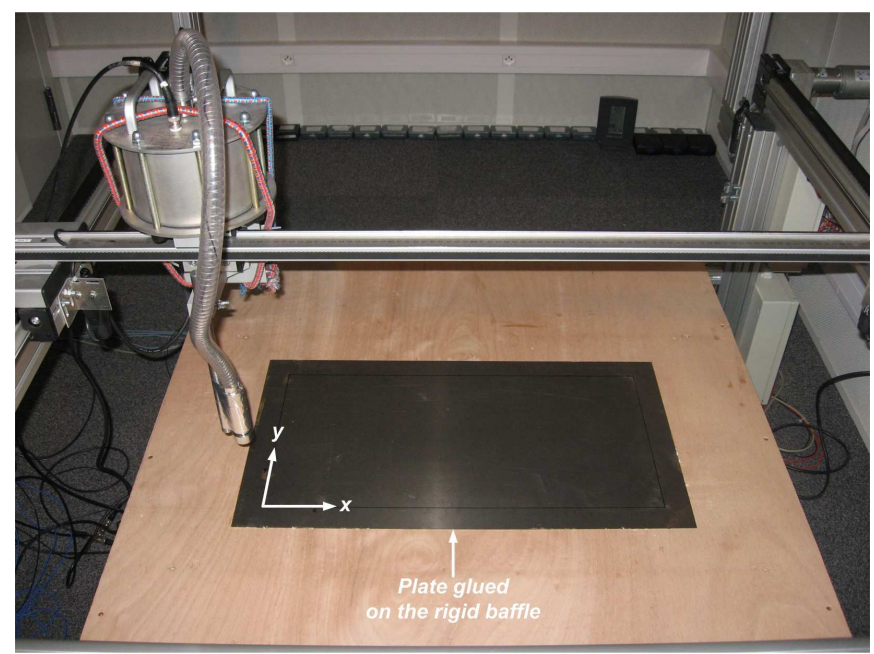

Figure 16: Experimental set-up for measuring the transfer functions $H_{Q m}$

Fig. 17. The modal behaviour of the plate can be seen clearly.

Finally, it is important to notice that the experimental measurement of $H_{Q m}$ takes 30 minutes for each point $\mathrm{Q}$. As a consequence, the use of an accelerometer is quite cumbersome to evaluate the whole behaviour of the structure from its vibration power-spectral density function for instance. To by pass this difficulty, non contact techniques, such as scanning laser vibrometers or digital image correlation methods [31, 32], can be used instead.

\subsection{Validation of the basic principle}

Two validation steps are presented in this section: (a) Reconstructions of wall pressure plane waves and (b) Comparison with a classical random vibration formulation when the transfer functions $H_{Q m}$ are derived from a semi-analytical calculation. TBL excitation is described by the Corcos model [see Eq. (16)] whose parameters are given in table 1. The TBL wall pressure 


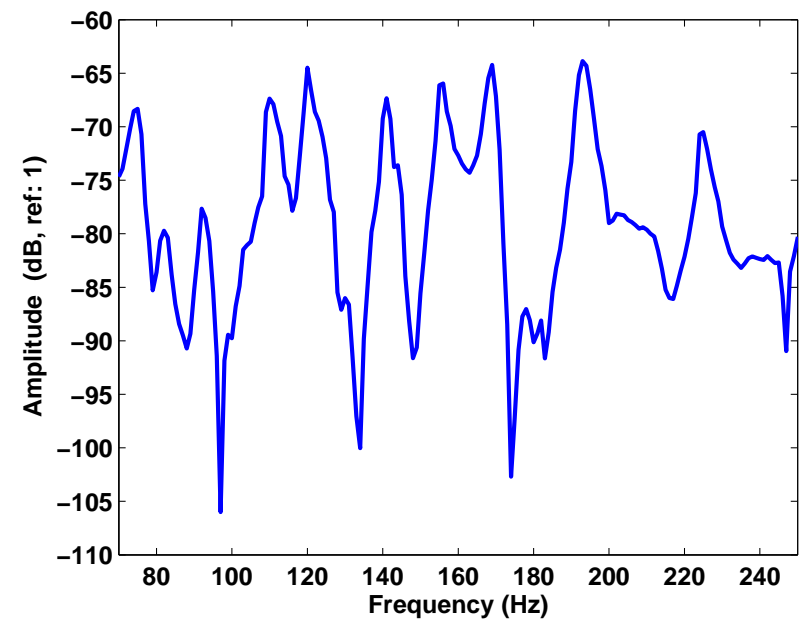

Figure 17: Typical result of $H_{Q m}$ measurements - Modulus of the transfer function $\left|H_{Q m}\right|$ measured between the particle velocity of the monopole-like source and the induced structural velocity of a steel plate of dimensions $0.6 \times 0.3 \times 0.0005 \mathrm{~m}^{3}$

frequency spectrum associated with the Corcos model is that measured by Totaro et al. in a wind tunnel [33] and presented in Fig. 18. Nevertheless, the Corcos model is only considered as an example, since other models can be used by adapting the ASD function $S_{A_{r s} A_{r s}}(\omega)$ to the chosen model.

\subsubsection{Reconstruction of wall pressure plane waves}

In this section, a wall pressure plane wave of unit amplitude is compared to that reconstructed by using the synthetic array approach for two couples of wavenumbers $\left(k_{r}, k_{s}\right)=\left(4.5 \mathrm{~m}^{-1}, 4.5 \mathrm{~m}^{-1}\right)$ and $\left(k_{r}, k_{s}\right)=\left(20 \mathrm{~m}^{-1}, 20 \mathrm{~m}^{-1}\right)$.

Fig. 19 presents, for both couples of wavenumbers, the comparison of the wall pressure plane waves to be reconstructed and those reconstructed by 


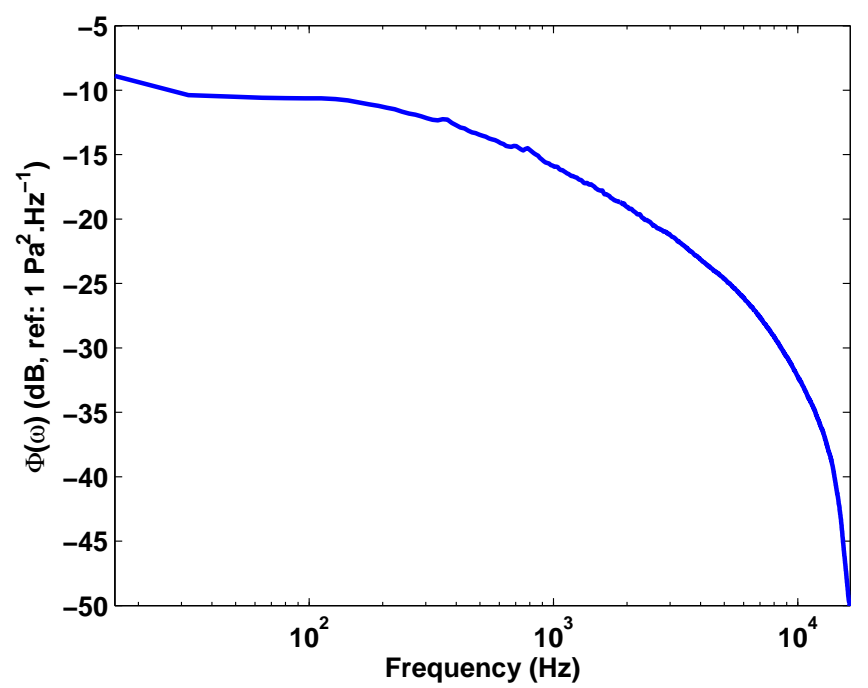

Figure 18: TBL wall pressure frequency spectrum measured in wind tunnel for the free stream velocity $U_{\infty}=50 \mathrm{~m} . \mathrm{s}^{-1}$, after Totaro et al. [33]

the synthetic array approach at $250 \mathrm{~Hz}$. This figure shows clearly that the amplitudes as well as the spatial variations of the wall pressure plane waves are well reproduced. Consequently, this validates the experimental technique proposed for reconstructing wall pressure plane waves.

\subsubsection{Semi-analytical validation of TBL induced vibration}

A semi-analytical validation of the synthetic array approach is proposed in this section. It consists in comparing the structural velocity ASD function of a simply supported plate calculated on the basis of the standard random theory with that obtained from the synthetic array approach. This calculation is an additional step for assessing the validity of the proposed approach. To this end, the reference calculation was carried out by using the discretized form of Eq. (18) [see Eq. (19)] over a grid of 231 discretization points in 


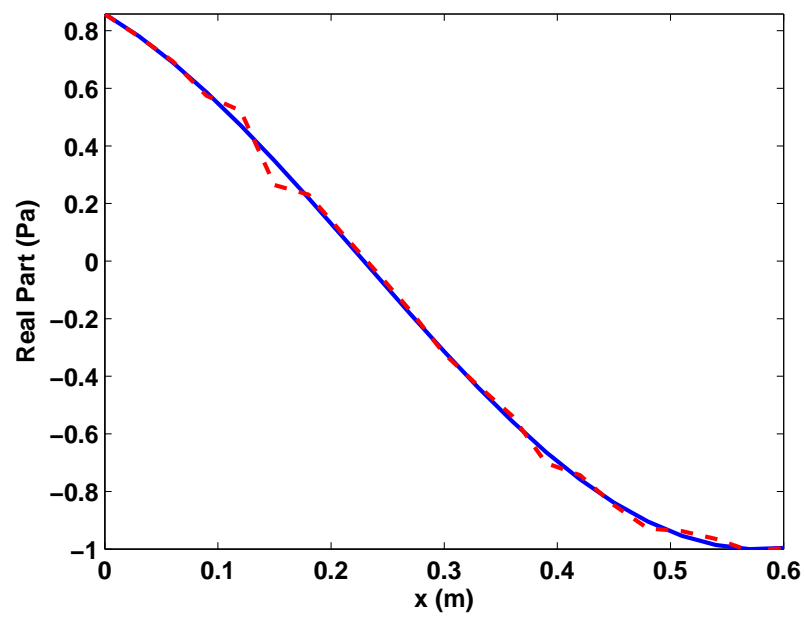

(a)

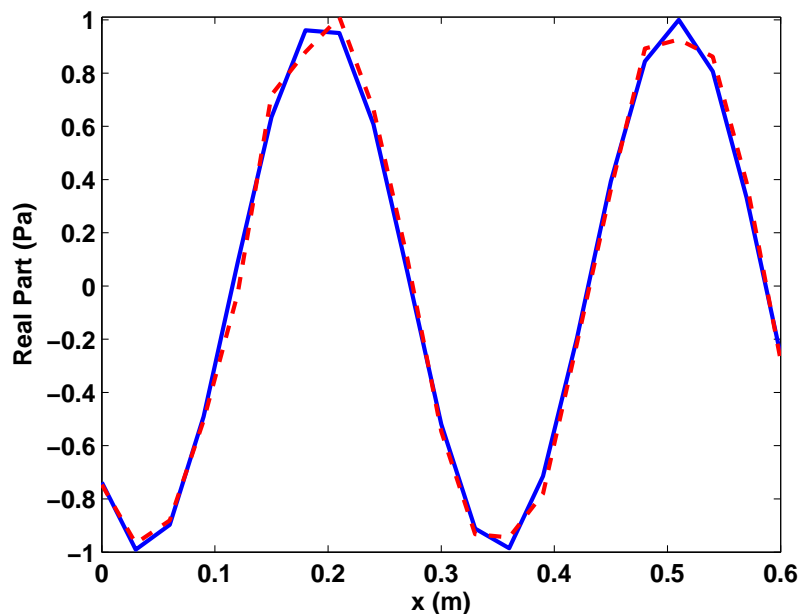

(b)

Figure 19: Reconstruction of wall pressure plane waves (a) for $\left(k_{r}, k_{s}\right)=$ $\left(4.5 \mathrm{~m}^{-1}, 4.5 \mathrm{~m}^{-1}\right)$ and (b) for $\left(k_{r}, k_{s}\right)=\left(20 \mathrm{~m}^{-1}, 20 \mathrm{~m}^{-1}\right)$ in the plane $(x, 0.12 \mathrm{~m})$ at $250 \mathrm{~Hz}-(-)$ wall pressure plane wave to be reconstructed (Reference) and (--) wall pressure plane wave reconstructed by the synthetic array approach 
order to ensure consistency with the observation mesh used for measuring the transfer functions $H_{m p}$.

$$
S_{v v}(Q, \omega)=\iint_{S_{p}} H_{v}^{*}(Q, M, \omega) S_{p p}\left(Q, M^{\prime}, \omega\right) H_{v}\left(Q, M^{\prime}, \omega\right) \mathrm{d} M \mathrm{~d} M^{\prime}
$$

where $S_{p p}\left(Q, M^{\prime}, \omega\right)$ is the TBL CSD function, $H_{v}(Q, M, \omega)$ the transfer function corresponding to the structural velocity at point $\mathrm{Q}$ when the plate is excited by a point force at point $\mathrm{M}$ and $H_{v}^{*}$ the complex conjugate of $H_{v}$.

$$
S_{v v}(Q, \omega)=\sum_{i} \sum_{j} H_{v}^{*}\left(Q, M_{i}, \omega\right) S_{p p}\left(M_{i}, M_{j}, \omega\right) H_{v}\left(Q, M_{j}, \omega\right) \Delta M_{i} \Delta M_{j}
$$

where $\Delta M_{i}$, resp. $\Delta M_{j}$, is the spatial resolution.

Then, the calculation of the structural velocity ASD function by the synthetic array approach is derived from Eqs. (9) and (17), where the transfer functions $H_{Q m}$ are semi-analytically computed from Eq. (20) and correspond to the response of the plate to a monopole source of unit amplitude.

$$
H_{Q m}(\omega)=j \omega \sum_{n} \frac{P_{n}^{m} \phi_{n}(Q)}{M_{n}\left(\omega_{n}^{2}-\omega^{2}+j \eta_{n} \omega \omega_{n}\right)}
$$

where $M_{n}$ is the generalized mass, $\omega_{n}$ the natural frequency of the plate, $\phi_{n}$ the mode shape, $\eta_{n}$ the modal damping. The generalized force $P_{n}^{m}$ is given by the following equation:

$$
P_{n}^{m}=\int_{S_{p}} H(\omega) \phi_{n}(P) d S_{p} \approx \sum_{p} H_{m p}(\omega) \phi_{n}^{p} \Delta S_{p}
$$


where $H_{m p}(\omega)$ are the measured transfer functions, $H(\omega)$ is the continuous form of $H_{m p}(\omega), \phi_{n}^{p}$ the mode shapes of the plate discretized over the $\mathrm{p}$ observation points and $\Delta S_{p}$ a surface element.

Fig. 20 presents a comparison of the structural velocity ASD function at point $\mathrm{Q}(0.22 \mathrm{~m}, 0.23 \mathrm{~m})$ of a $0.6 \times 0.3 \times 0.0005 \mathrm{~m}^{3}$ simply supported plate, obtained either from the classical random vibration formulation given by Eq. (8) or assessed by the synthetic array approach from Eqs. (13), (20), (17) and (9). As expected, good agreement between both results is observed, despite discrepancies of $3 \mathrm{~dB}$ in some frequency bands of low level, which is not crucial, since the vibration levels at the resonance frequencies are well estimated. Thus this result allows validating the experimental technique developed in this paper to characterize the vibrations induced by TBL.

\section{Versatility of the synthetic array approach}

As mentioned in the section 2, numerous models are available in the literature to represent TBL wall pressure fluctuations on a rigid plane. These models link wall pressure fluctuations to the main features of the flow (convection velocity, boundary layer thickness, ... ). One of the main features of the synthetic array approach is its versatility with respect to the TBL excitation model $\Gamma_{p p}\left(k_{r}, k_{s}, \omega\right)$, since the technique proposed is based on discrete wave-vector integration models. Consequently, all the TBL models expressed in the wavenumber-frequency space can be used. Furthermore, the comparison of these models can be carried out in a straightforward manner, since the introduction of TBL excitation is performed in the numerical stages by 


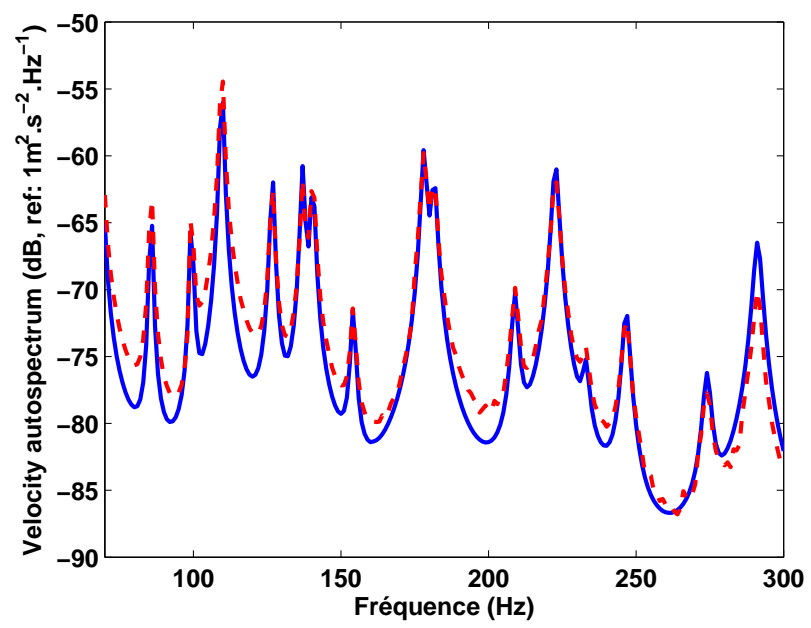

Figure 20: Comparison of the structural velocity auto-spectral density function $S_{v v}(Q, \omega)$ at point $\mathrm{Q}(0.22 \mathrm{~m}, 0.23 \mathrm{~m})$ computed by $(-)$ the classical random vibration formulation (Reference) and (--) the synthetic array approach by using the measurements of $H_{m p}$ and a semi-analytical calculation of $H_{Q m}$ 
using Eqs. (13), (17) and (9).

To demonstrate the ability of our experimental technique to deal with different TBL excitation models, full implementation of the procedure is proposed, that is to say that measurements of $H_{m p}$ and $H_{Q m}$ are used to evaluate the TBL induced vibrations of the steel plate defined in section 5 . In the following discussion, TBL models proposed by Corcos [17], Efimtsov [18], Chase [22] and Smol'yakov and Tkachenko [20] are compared for a fully developed turbulent flow whose parameters were measured in a wind tunnel by Totaro et al. [33] (see table 4). Fig. 21 presents the wavenumber-frequency spectrum predicted by the 4 models. For the sake of brevity, these models are not presented here, but detailed reviews and discussions about TBL models can be found in Refs. [24], [34] and [35]. Finally, the parameters of the TBL models used in this article are those proposed by their authors, except for the Corcos model, whose parameters are defined in table 1.

Table 4: Turbulent flow parameters

\begin{tabular}{ll}
\hline Parameters & Values \\
\hline Convection velocity & $U_{c}=35 \mathrm{~m} . \mathrm{s}^{-1}$ \\
Friction velocity & $u_{\tau}=1.96 \mathrm{~m} . \mathrm{s}^{-1}$ \\
Boundary layer thickness & $\delta=85 \mathrm{~mm}$ \\
Displacement thickness & $\delta^{*}=8.8 \mathrm{~mm}$ \\
Momentum thickness & $\theta=6.7 \mathrm{~mm}$ \\
Wall shear stress & $\tau_{w}=4.58 \mathrm{~Pa}$ \\
\hline
\end{tabular}




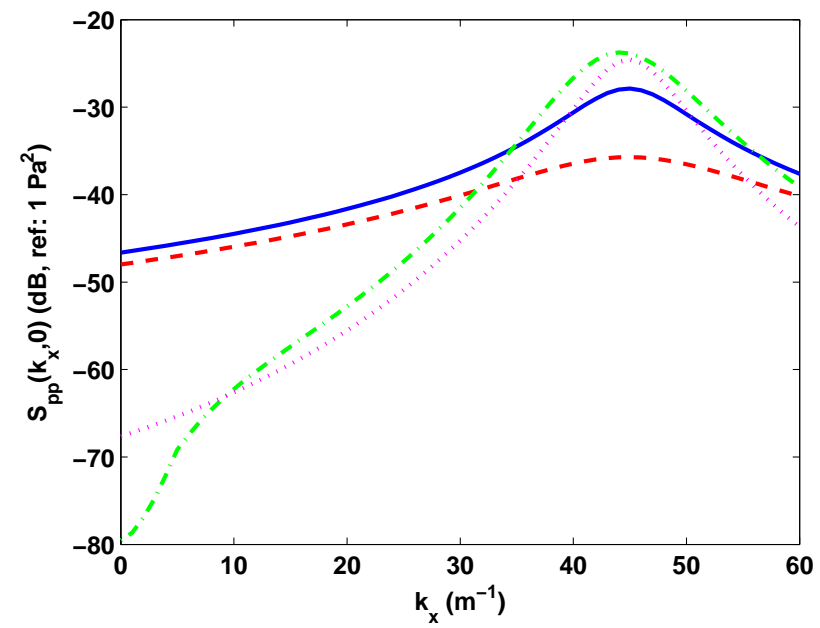

Figure 21: Comparison of TBL wavenumber-frequency spectra at $250 \mathrm{~Hz}-(-)$ Corcos model, (--) Efimtsov model, (-.-) Chase model and (...) Smol'yakov \& Tkachenko model

The comparison of the structural velocity $\mathrm{ASD}$ function $S_{v v}(Q, \omega)$ at point Q $(0.22 \mathrm{~m}, 0.23 \mathrm{~m})$ of the plate, evaluated from the synthetic array approach for the 4 TBL excitation models mentioned above, is presented in Fig. 22. Observation of these results brings to light the fact that they are clustered together within $6 \mathrm{~dB}$ at most throughout the frequency range, despite large differences of TBL wall pressure spectra in the low-wavenumber region. These results are consistent with previous investigations into TBL induced vibrations $[35,36,37]$. Indeed, plate bending waves are only excited by the TBL pressure components of the matching scales and, in the present case, the bending waves are predominantly excited by convective components, since the frequency range of interest is below the aerodynamic coincidence frequency $(\approx 250 \mathrm{~Hz})$ for the plate under consideration. Consequently, the 
differences observed in Fig. 22 can be explained for the most part by the modeling of the convective components in the 4 TBL models, as shown in Fig. 21.

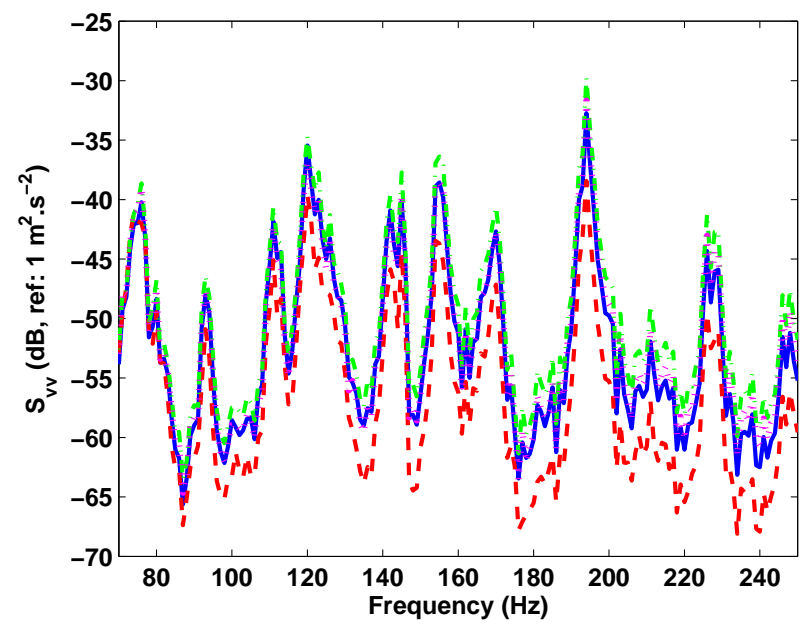

Figure 22: Comparison of structural velocity auto-spectral density function $S_{v v}(Q, \omega)$ at point Q $(0.22 \mathrm{~m}, 0.23 \mathrm{~m})$ for various TBL excitation models - (-) Corcos model, $(--)$ Efimtsov model, (-.-) Chase model and (...) Smol'yakov \& Tkachenko model

\section{Conclusion}

In the present study, the development of a general methodology to simulate experimentally the response of linear structures excited by stationary random pressure fields was investigated. Here, the TBL excitation was chosen as an example. The method proposed relies on the experimental simulation of a set of uncorrelated wall pressure plane waves, whose amplitudes are scaled on the TBL CSD function expressed in the wavenumber space. This can be 
achieved with an array of acoustic monopole-like sources. The main characteristics of the monopole array were determined by performing a numerical parametric study. In particular, it was shown that the number of acoustic monopoles increases with frequency. This would lead to an unrealistic density of sources if considering a complete array. To overcome this difficulty, the linearity of the problem was used to replace the monopole array with a synthetic array made up of a single monopole moved in space.

Such a methodology allows a higher flexibility of the experimental set-up regarding the total number of monopoles required to suitably reconstruct wall pressure plane waves. From the practical standpoint, dispersions of sensor characteristics are avoided and only transfer function measurements are required. The counterpart of the sequential nature of the simulation process is the need of a precise source positioning to avoid phase shifts, as well as the need of a stable environment to avoid variations of the experimental set-up with time.

From these technical requirements, an experimental set-up was designed to validate the approach proposed under laboratory conditions. The results obtained on an academic structure show the method is capable of reconstructing wall pressure plane waves and the structural velocity ASD function of a plate subjected to TBL excitation. Finally, the versatility of the synthetic array approach with respect to TBL excitation models was highlighted. A test bench using a robot and a laser vibrometer should be developed in order to apply the methodology detailed in this paper to industrial structures. 


\section{Acknowledgments}

The authors are grateful to the French Ministry of Defence for funding this work.

\section{References}

[1] W. V. Bhat. Flight test measurement of exterior turbulent boundary layer pressure fluctuations on boeing model 737 airplane. Journal of Sound and Vibration, 14 (4):439-457, 1971.

[2] J. F. Wilby and F. L. Gloyna. Vibration measurements of an airplane fuselage structure, part I: Turbulent boundary excitation. Journal of Sound and Vibration, 23 (4):443-466, 1972.

[3] J. Sulc, J. Hofr, and L. Benda. Exterior noise on the fuselage of light propellor aircraft in flight. Journal of Sound and Vibration, 84 (1):105$120,1982$.

[4] R. Rackl and A. Weston. Modeling of turbulent boundary layer surface pressure fluctuation auto and cross spectra: Verification and adjustments based on tu-144ll data. Technical report, NASA, 2005.

[5] R. A. Katz, T. A. Galib, and J. Cembrola. Mechanisms underlying transitional and turbulent boundary layer (TBL) flow-induced noise in underwater acoustics. Journal de Physique IV, Colloque C5, supplément au Journal de Physique III, 4:1063-1066, 1994. 
[6] T. A. Galib, R. A. Katz, S. H. Ko, and B. Sandman. Measurements of turbulent pressure fluctuations using a buoyant vehicle coated with a thin elastomer layer. J. Acoust. Soc. Am., 96 (6):3800-3803, 1994.

[7] T. M. Farabee and M. J. Casarella. Spectral features of wall pressure fluctuations beneath turbulent boundary layers. Physics of Fluids A, 3:2410-2420, 1991.

[8] S. Finnveden, F. Birgersson, U. Ross, and T. Kremer. A model of wall pressure correlation for prediction of turbulence-induced viration. Journal of Fluids and Structures, 20:1127-1143, 2005.

[9] F. J. Fahy. On simulating the transmission through structures of noise from turbulent boundary layer pressure fluctuations. Journal of Sound and Vibration, 3 (1):57-81, 1966.

[10] G. Robert. Modélisation et simulation du champ excitateur induit sur une structure par une couche limite turbulente (Modelling and simulation of the induced exciting field on a structure by a turbulent boundary layer). Phd thesis, Ecole Centrale de Lyon, 1984.

[11] S. J. Elliott, C. Maury, and P. Gardonio. The synthesis of spatially correlated random pressure fields. J. Acoust. Soc. Am., 117 (3):11861201, 2005.

[12] S. H. Crandall. Random vibration: Volume 1, chapter Statistical properties of response to random vibration, pages 77-90. MIT Press, 1959.

[13] J. Audet, M. Lagier, P. Marin-Curtoud, and T. Rohan. Turbulent 
boundary layer simulation setup on a sonar dome. J. Acoust. Soc. Am., 84 (S1):S118-S119, 1988.

[14] T. Bravo and C. Maury. The experimental synthesis of random pressure fields: Methodology. J. Acoust. Soc. Am., 120 (5):2702-2711, 2006.

[15] C. Maury and T. Bravo. The experimental synthesis of random pressure fields: Practical feasability. J. Acoust. Soc. Am., 120 (5):2712-2723, 2006.

[16] T. Bravo and C. Maury. A synthesis approach for reproducing the response of arircraft panels to a turbulent boundary layer excitation. $J$. Acoust. Soc. Am., 129 (1):143-153, 2011.

[17] G. M. Corcos. Resolution of pressure in turbulence. J. Acoust. Soc. Am., 35 (2):192-199, 1963.

[18] B. M. Efimtsov. Characteristics of the field of turbulent wall pressure fluctuations at large reynolds numbers. Soviet Physics Acoustics, 28 (4):289-292, 1982.

[19] Y. F. Hwang and F. E. Geib. Estimation of the wavevector-frequency spectrum of turbulent boundary layer wall pressure by mutiple linear regression. Journal of Vibration, Acoustics, Stress and Reliability in Design - Transactions of the ASME, 106:334-3442, 1984.

[20] A. V. Smol'yakov and V. M. Tkachenko. Model of a field of pseudosonic turbulent wall pressures and experimental data. Soviet Physics Acoustics, 37 (6):627-631, 1991. 
[21] D. M. Chase. Modeling the wavevector-frequency spectrum of turbulent boundary layer wall pressure. Journal of Sound and Vibration, 70:29-67, 1980.

[22] D. M . Chase. The character of turbulent wall pressure spectrum at subconvective wavenumbers and a suggested comprehensive model. Journal of Sound and Vibration, 112 (1):127-147, 1987.

[23] M. S. Howe. Surface pressures and sound produced by turbulent flow over smooth and rough walls. J. Acoust. Soc. Am., 90 (2):1041-1047, 1991.

[24] Y. F. Hwang, W. K. Bonness, and S. A. Hambric. On modeling structural excitations by low speed turbulent boundary layer flows. Technical report, Applied Research Laboratory - Pennstate University, 2003.

[25] A. Powell. Random vibration: Volume 1, chapter On the response of structures to random pressures and to jet noise in particular, pages 187-229. MIT Press, 1959.

[26] W. A. Strawderman and R. S. Brand. Turbulent-flow-excited vibration of a simply supported, rectangular flat plate. J. Acoust. Soc. Am., 45 (1):177-192, 1969.

[27] A. D. Pierce. Acoustics: An Introduction to Its Physical Principles and Applications. Acoustical Society of America, New York, 1981.

[28] C. Maury, P. Gardonio, and S. J. Elliott. A wavenumber approach to modelling the response of a randomly excited panel, part i: general theory. Journal of Sound and Vibration, 252 (1):83-113, 2002. 
[29] Y. F. Hwang and G. Maidanik. A wavenumber analysis of the coupling of a structural mode and flow turbulence. Journal of Sound and Vibration, 142 (1):135-152, 1990.

[30] S. W. Autrey. Passive synthetic array. J. Acoust. Soc. Am., 84 (2):592598, 1988.

[31] M. A. Sutton, W. J. Wolters, W. H. Peters, W.F. Ranson, and S.R. McNeill. Determination of displacement using an improved digital correlation method. Image and Vision Computing, 1 (3):133-139, 1983.

[32] M. N. Helfrick, C. Niezrecki, P. Avitabile, and T. Schmidt. 3d digital image correlation methods for full-field vibration measurement. Mechanical Systems and Signal Processing, 25 (3):917-927, 2011.

[33] N. Totaro, G. Robert, and J-L. Guyader. Frequency averaged injected power under boundary layer excitation : An experimental validation. Acta acustica united with acustica, 94 (4):534-547, 2008.

[34] M. K. Bull. Wall-pressure fluctuations beneath turbulent boundary layer: Some reflections on forty years of research. Journal of Sound and Vibration, 190 (3):299-315, 1996.

[35] W. R. Graham. A comparison of models for the wavenumber-frequency spectrum of turbulent boundary layer pressures. Journal of Sound and Vibration, 206 (4):541-565, 1997.

[36] A. O. Borisyuk and V. T. Grinchenko. Vibration and noise generation by elastic elements excited by turbulent flow. Journal of Sound and Vibration, 204 (2):213-237, 1997. 
[37] C. Maury, P. Gardonio, and S. J. Elliott. A wavenumber approach to modelling the response of a randomly excited panel, part ii: application to aircraft panels excited by a turbulent boundary layer. Journal of Sound and Vibration, 252 (1):115-139, 2002. 


\section{List of Figures}

1 Comparison of CSD function of a TBL and a diffuse field . . . 11

2 Definition of the array of acoustic monopoles . . . . . . . . . . 12

3 Influence of the number of acoustic monopoles on the reconstruction of a wall pressur

4 Influence of the number of acoustic monopoles on the reconstruction of the TBL CSD

5 Influence of the distance of the array to the observation area on the reconstruction of

6 Influence of the distance of the array to the observation area on the reconstruction of

$7 \quad$ Influence of the size of the monopole array . . . . . . . . . . . 23

8 Influence of the size of the monopole array on the reconstruction of the TBL CSD fur

$9 \quad$ Example of measurement of the transfer functions $H_{m p} \ldots \ldots 27$

10 Example of measurement of the transfer functions $H_{Q m}$. . . 28

11 Block diagram of the proposed experimental procedure . . . . 29

12 Principle of aerodynamic coincidence . . . . . . . . . . . . 31

13 Monopole-like source . . . . . . . . . . . . . . . . . 35

14 Experimental set-up for measuring the transfer functions $H_{m p} \quad 36$

15 Typical result of $H_{m p}$ measurements . . . . . . . . . . . . . 37

16 Experimental set-up for measuring the transfer functions $H_{Q m} \quad 38$

17 Typical result of $H_{Q m}$ measurements . . . . . . . . . . . . . 39

18 TBL wall pressure frequency spectrum . . . . . . . . . . . . . 40

19 Reconstruction of wall pressure plane waves . . . . . . . . . . 41

20 Comparison of the structural velocity ASD function $S_{v v}(Q, \omega) \quad 44$

21 Comparison of TBL wavenumber-frequency spectra at $250 \mathrm{~Hz} \quad 46$

22 Comparison of structural velocity ASD function $S_{v v}(Q, \omega)$ for various TBL models 47 